DESY-00-145

ISSN 0418-9833

October 2000

\title{
Measurement and QCD Analysis of Jet Cross Sections in Deep-Inelastic Positron-Proton Collisions at $\sqrt{s}$ of $300 \mathrm{GeV}$
}

\author{
H1 Collaboration
}

\begin{abstract}
Jet production is studied in the Breit frame in deep-inelastic positron-proton scattering over a large range of four-momentum transfers $5<Q^{2}<15000 \mathrm{GeV}^{2}$ and transverse jet energies $7<E_{T}<60 \mathrm{GeV}$. The analysis is based on data corresponding to an integrated luminosity of $\mathcal{L}_{\text {int }} \simeq 33 \mathrm{pb}^{-1}$ taken in the years 1995-1997 with the H1 detector at HERA at a center-of-mass energy $\sqrt{s}=300 \mathrm{GeV}$. Dijet and inclusive jet cross sections are measured multi-differentially using $k_{\perp}$ and angular ordered jet algorithms. The results are compared to the predictions of perturbative QCD calculations in next-to-leading order in the strong coupling constant $\alpha_{s}$. QCD fits are performed in which $\alpha_{s}$ and the gluon density in the proton are determined separately. The gluon density is found to be in good agreement with results obtained in other analyses using data from different processes. The strong coupling constant is determined to be $\alpha_{s}\left(M_{Z}\right)=0.1186 \pm 0.0059$. In addition an analysis of the data in which both $\alpha_{s}$ and the gluon density are determined simultaneously is presented.
\end{abstract}

To be submitted to Eur. Phys. J. C 
C. Adloff ${ }^{33}$, V. Andreev ${ }^{24}$, B. Andrieu ${ }^{27}$, T. Anthonis ${ }^{4}$, V. Arkadov ${ }^{35}$, A. Astvatsatourov ${ }^{35}$, I. Ayyaz ${ }^{28}$, A. Babaev ${ }^{23}$, J. Bähr ${ }^{35}$, P. Baranov ${ }^{24}$, E. Barrelet ${ }^{28}$, W. Bartel ${ }^{10}$, U. Bassler ${ }^{28}$, P. Bate ${ }^{21}$, A. Beglarian ${ }^{34}$, O. Behnke ${ }^{13}$, C. Beier ${ }^{14}$, A. Belousov ${ }^{24}$, T. Benisch ${ }^{10}$, Ch. Berger ${ }^{1}$, G. Bernardi ${ }^{28}$, T. Berndt ${ }^{14}$, J.C. Bizot ${ }^{26}$, V. Boudry ${ }^{27}$, W. Braunschweig ${ }^{1}$, V. Brisson ${ }^{26}$, H.-B. Bröker ${ }^{2}$, D.P. Brown ${ }^{11}$, W. Brückner ${ }^{12}$, P. Bruel ${ }^{27}$, D. Bruncko ${ }^{16}$, J. Bürger ${ }^{10}$, F.W. Büsser ${ }^{11}$, A. Bunyatyan ${ }^{12,34}$, H. Burkhardt ${ }^{14}$, A. Burrage ${ }^{18}$, G. Buschhorn ${ }^{25}$, A.J. Campbell ${ }^{10}$, J. Cao ${ }^{26}$, T. Carli ${ }^{25}$, S. Caron ${ }^{1}$, E. Chabert ${ }^{22}$, D. Clarke ${ }^{5}$, B. Clerbaux ${ }^{4}$, C. Collard ${ }^{4}$, J.G. Contreras ${ }^{7,41}$, Y.R. Coppens ${ }^{3}$, J.A. Coughlan ${ }^{5}$, M.-C. Cousinou ${ }^{22}$, B.E. Cox ${ }^{21}$, G. Cozzika ${ }^{9}$, J. Cvach $^{29}$, J.B. Dainton ${ }^{18}$, W.D. Dau ${ }^{15}$, K. Daum ${ }^{33,39}$,

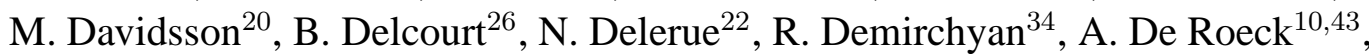
E.A. De Wolf ${ }^{4}$, C. Diaconu ${ }^{22}$, P. Dixon ${ }^{19}$, V. Dodonov ${ }^{12}$, J.D. Dowell ${ }^{3}$, A. Droutskoi ${ }^{23}$, C. Duprel ${ }^{2}$, G. Eckerlin ${ }^{10}$, D. Eckstein ${ }^{35}$, V. Efremenko ${ }^{23}$, S. Egli ${ }^{32}$, R. Eichler ${ }^{36}$, F. Eisele ${ }^{13}$, E. Eisenhandler ${ }^{19}$, M. Ellerbrock ${ }^{13}$, E. Elsen ${ }^{10}$, M. Erdmann ${ }^{10,40, e}$, W. Erdmann ${ }^{36}$, P.J.W. Faulkner ${ }^{3}$, L. Favart ${ }^{4}$, A. Fedotov ${ }^{23}$, R. Felst ${ }^{10}$, J. Ferencei ${ }^{10}$, S. Ferron ${ }^{27}$, M. Fleischer ${ }^{10}$, Y.H. Fleming ${ }^{3}$, G. Flügge ${ }^{2}$, A. Fomenko ${ }^{24}$, I. Foresti ${ }^{37}$, J. Formánek ${ }^{30}$, J.M. Foster ${ }^{21}$, G. Franke ${ }^{10}$, E. Gabathuler ${ }^{18}$, K. Gabathuler ${ }^{32}$, J. Garvey $^{3}$, J. Gassner ${ }^{32}$, J. Gayler ${ }^{10}$, R. Gerhards ${ }^{10}$, S. Ghazaryan ${ }^{34}$, L. Goerlich ${ }^{6}$, N. Gogitidze ${ }^{24}$, M. Goldberg ${ }^{28}$, C. Goodwin ${ }^{3}$, C. Grab ${ }^{36}$, H. Grässler ${ }^{2}$, T. Greenshaw ${ }^{18}$, G. Grindhammer ${ }^{25}$, T. Hadig ${ }^{13}$, D. Haidt ${ }^{10}$, L. Hajduk ${ }^{6}$, W.J. Haynes ${ }^{5}$, B. Heinemann ${ }^{18}$, G. Heinzelmann ${ }^{11}$, R.C.W. Henderson ${ }^{17}$, S. Hengstmann ${ }^{37}$, H. Henschel ${ }^{35}$, R. Heremans ${ }^{4}$, G. Herrera ${ }^{7,41}$, I. Herynek ${ }^{29}$, M. Hildebrandt ${ }^{37}$, M. Hilgers ${ }^{36}$, K.H. Hiller ${ }^{35}$, J. Hladký ${ }^{29}$, P. Höting ${ }^{2}$, D. Hoffmann ${ }^{10}$, W. Hoprich ${ }^{12}$, R. Horisberger ${ }^{32}$, S. Hurling ${ }^{10}$, M. Ibbotson ${ }^{21}$, Ç. İşsever ${ }^{7}$, M. Jacquet ${ }^{26}$, M. Jaffre ${ }^{26}$, L. Janauschek ${ }^{25}$, D.M. Jansen ${ }^{12}$, X. Janssen ${ }^{4}$, V. Jemanov ${ }^{11}$, L. Jönsson ${ }^{20}$, D.P. Johnson ${ }^{4}$, M.A.S. Jones ${ }^{18}$, H. Jung ${ }^{20}$, H.K. Kästli ${ }^{36}$, D. Kant ${ }^{19}$, M. Kapichine ${ }^{8}$, M. Karlsson ${ }^{20}$, O. Karschnick ${ }^{11}$, F. Keil ${ }^{14}$, N. Keller ${ }^{37}$, J. Kennedy ${ }^{18}$, I.R. Kenyon ${ }^{3}$, S. Kermiche ${ }^{22}$, C. Kiesling ${ }^{25}$, M. Klein ${ }^{35}$, C. Kleinwort ${ }^{10}$, G. Knies ${ }^{10}$, B. Koblitz ${ }^{25}$, S.D. Kolya ${ }^{21}$, V. Korbel ${ }^{10}$, P. Kostka ${ }^{35}$, S.K. Kotelnikov ${ }^{24}$, R. Koutouev ${ }^{12}$, A. Koutov ${ }^{8}$, M.W. Krasny ${ }^{28}$, H. Krehbiel ${ }^{10}$, J. Kroseberg ${ }^{37}$, K. Krüger ${ }^{10}$, A. Küpper ${ }^{33}$, T. Kuhr ${ }^{11}$, T. Kurča ${ }^{35,16}$, R. Lahmann ${ }^{10}$, D. Lamb ${ }^{3}$, M.P.J. Landon ${ }^{19}$, W. Lange ${ }^{35}$, T. Laštovička ${ }^{30}$, E. Lebailly ${ }^{26}$, A. Lebedev ${ }^{24}$, B. Leißner ${ }^{1}$, R. Lemrani ${ }^{10}$, V. Lendermann ${ }^{7}$, S. Levonian ${ }^{10}$, M. Lindstroem ${ }^{20}$, B. List ${ }^{36}$, E. Lobodzinska ${ }^{10,6}$, B. Lobodzinski ${ }^{6,10}$, A. $\operatorname{Loginov}^{23}$, N. Loktionova ${ }^{24}$, V. Lubimov ${ }^{23}$, S. Lüders ${ }^{36}$, D. Lüke ${ }^{7,10}$, L. Lytkin ${ }^{12}$, N. Magnussen ${ }^{33}$, H. Mahlke-Krüger ${ }^{10}$, N. Malden ${ }^{21}$, E. Malinovski ${ }^{24}$, I. Malinovski $^{24}$, R. Maraček ${ }^{25}$, P. Marage ${ }^{4}$, J. Marks ${ }^{13}$, R. Marshall ${ }^{21}$, H.-U. Martyn ${ }^{1}$, J. Martyniak ${ }^{6}$, S.J. Maxfield ${ }^{18}$, A. Mehta ${ }^{18}$, K. Meier ${ }^{14}$, P. Merkel ${ }^{10}$, F. Metlica ${ }^{12}$, A.B. Meyer ${ }^{11}$, H. Meyer ${ }^{33}$, J. Meyer ${ }^{10}$, P.-O. Meyer ${ }^{2}$, S. Mikocki ${ }^{6}$, D. Milstead ${ }^{18}$, T. Mkrtchyan ${ }^{34}$, R. Mohr ${ }^{25}$, S. Mohrdieck ${ }^{11}$, M.N. Mondragon ${ }^{7}$, F. Moreau ${ }^{27}$, A. Morozov ${ }^{8}$, J.V. Morris ${ }^{5}$, K. Müller ${ }^{13}$, P. Murín ${ }^{16,42}$, V. Nagovizin ${ }^{23}$, B. Naroska ${ }^{11}$, J. Naumann ${ }^{7}$, Th. Naumann ${ }^{35}$, G. Nellen ${ }^{25}$, P.R. Newman ${ }^{3}$, T.C. Nicholls ${ }^{5}$, F. Niebergall ${ }^{11}$, C. Niebuhr ${ }^{10}$, O. Nix ${ }^{14}$, G. Nowak ${ }^{6}$, T. Nunnemann ${ }^{12}$, J.E. Olsson ${ }^{10}$, D. Ozerov ${ }^{23}$, V. Panassik ${ }^{8}$, C. Pascaud $^{26}$, G.D. Patel ${ }^{18}$, E. Perez ${ }^{9}$, J.P. Phillips ${ }^{18}$, D. Pitzl ${ }^{10}$, R. Pöschl ${ }^{7}$, I. Potachnikova ${ }^{12}$, B. Povh ${ }^{12}$, K. Rabbertz ${ }^{1}$, G. Rädel ${ }^{9}$, J. Rauschenberger ${ }^{11}$, P. Reimer ${ }^{29}$, B. Reisert ${ }^{25}$, D. Reyna ${ }^{10}$, S. Riess ${ }^{11}$, C. Risler ${ }^{25}$, E. Rizvi ${ }^{3}$, P. Robmann ${ }^{37}$, R. Roosen ${ }^{4}$, A. Rostovtsev ${ }^{23}$, C. Royon ${ }^{9}$, S. Rusakov ${ }^{24}$, K. Rybicki ${ }^{6}$, D.P.C. Sankey ${ }^{5}$, J. Scheins ${ }^{1}$, F.-P. Schilling ${ }^{13}$, P. Schleper ${ }^{13}$, D. Schmidt ${ }^{33}$, D. Schmidt ${ }^{10}$, S. Schmitt ${ }^{10}$, L. Schoeffel ${ }^{9}$, A. Schöning ${ }^{36}$, T. Schörner ${ }^{25}$, V. Schröder ${ }^{10}$, H.-C. Schultz-Coulon ${ }^{7}$, C. Schwanenberger ${ }^{10}$, K. Sedlák ${ }^{29}$, F. Sefkow ${ }^{37}$, V. Shekelyan ${ }^{25}$, 
I. Sheviakov ${ }^{24}$, L.N. Shtarkov ${ }^{24}$, G. Siegmon ${ }^{15}$, P. Sievers ${ }^{13}$, Y. Sirois ${ }^{27}$, T. Sloan ${ }^{17}$, P. Smirnov ${ }^{24}$, V. Solochenko ${ }^{23, \dagger}$, Y. Soloviev ${ }^{24}$, V. Spaskov ${ }^{8}$, A. Specka ${ }^{27}$, H. Spitzer ${ }^{11}$, R. Stamen ${ }^{7}$, J. Steinhart ${ }^{11}$, B. Stella ${ }^{31}$, A. Stellberger ${ }^{14}$, J. Stiewe ${ }^{14}$, U. Straumann ${ }^{37}$, W. Struczinski ${ }^{2}$, M. Swart ${ }^{14}$, M. Taševský ${ }^{29}$, V. Tchernyshov ${ }^{23}$, S. Tchetchelnitski ${ }^{23}$, G. Thompson ${ }^{19}$, P.D. Thompson ${ }^{3}$, N. Tobien ${ }^{10}$, D. Traynor ${ }^{19}$, P. Truöl ${ }^{37}$, G. Tsipolitis ${ }^{10,38}$, I. Tsurin ${ }^{35}$, J. Turnau ${ }^{6}$, J.E. Turney ${ }^{19}$, E. Tzamariudaki ${ }^{25}$, S. Udluft ${ }^{25}$, A. Usik ${ }^{24}$, S. Valkár ${ }^{30}$, A. Valkárová ${ }^{30}$, C. Vallée ${ }^{22}$, P. Van Mechelen ${ }^{4}$, S. Vassiliev $^{8}$, Y. Vazdik $^{24}$, A. Vichnevski ${ }^{8}$, S. von Dombrowski ${ }^{37}$, K. Wacker ${ }^{7}$, R. Wallny ${ }^{37}$, T. Walter ${ }^{37}$, B. Waugh ${ }^{21}$, G. Weber ${ }^{11}$, M. Weber ${ }^{14}$, D. Wegener ${ }^{7}$, M. Werner ${ }^{13}$, G. White ${ }^{17}$, S. Wiesand ${ }^{33}$, T. Wilksen ${ }^{10}$, M. Winde ${ }^{35}$, G.-G. Winter ${ }^{10}$, C. Wissing ${ }^{7}$, M. Wobisch ${ }^{2}$, H. Wollatz ${ }^{10}$, E. Wünsch ${ }^{10}$, A.C. Wyatt ${ }^{21}$, J. Žáček ${ }^{30}$, J. Zálešák ${ }^{30}$, Z. Zhang ${ }^{26}$, A. Zhokin ${ }^{23}$, F. Zomer ${ }^{26}$, J. Zsembery ${ }^{9}$, and M. zur Nedden ${ }^{10}$

${ }^{1}$ I. Physikalisches Institut der RWTH, Aachen, Germany ${ }^{a}$

${ }^{2}$ III. Physikalisches Institut der RWTH, Aachen, Germany ${ }^{a}$

${ }^{3}$ School of Physics and Space Research, University of Birmingham, Birmingham, $U K^{b}$

${ }^{4}$ Inter-University Institute for High Energies ULB-VUB, Brussels; Universitaire Instelling Antwerpen, Wilrijk; Belgium ${ }^{c}$

${ }^{5}$ Rutherford Appleton Laboratory, Chilton, Didcot, $U K^{b}$

${ }^{6}$ Institute for Nuclear Physics, Cracow, Poland ${ }^{2}$

${ }^{7}$ Institut für Physik, Universität Dortmund, Dortmund, Germany ${ }^{a}$

${ }^{8}$ Joint Institute for Nuclear Research, Dubna, Russia

${ }^{9}$ CEA, DSM/DAPNIA, CE-Saclay, Gif-sur-Yvette, France

${ }^{10}$ DESY, Hamburg, Germany ${ }^{a}$

${ }^{11}$ II. Institut für Experimentalphysik, Universität Hamburg, Hamburg, Germany ${ }^{a}$

${ }^{12}$ Max-Planck-Institut für Kernphysik, Heidelberg, Germany ${ }^{a}$

${ }^{13}$ Physikalisches Institut, Universität Heidelberg, Heidelberg, Germany ${ }^{a}$

${ }^{14}$ Kirchhoff-Institut für Physik, Universität Heidelberg, Heidelberg, Germany ${ }^{a}$

${ }^{15}$ Institut für experimentelle und angewandte Kernphysik, Universität Kiel, Kiel, Germany ${ }^{a}$

${ }^{16}$ Institute of Experimental Physics, Slovak Academy of Sciences, Košice, Slovak Republic ${ }^{e, f}$

${ }^{17}$ School of Physics and Chemistry, University of Lancaster, Lancaster, UK ${ }^{b}$

${ }^{18}$ Department of Physics, University of Liverpool, Liverpool, $U K^{b}$

${ }^{19}$ Queen Mary and Westfield College, London, $U K^{b}$

${ }^{20}$ Physics Department, University of Lund, Lund, Sweden ${ }^{g}$

${ }^{21}$ Physics Department, University of Manchester, Manchester, $U K^{b}$

${ }^{22}$ CPPM, CNRS/IN2P3 - Univ Mediterranee, Marseille - France

${ }^{23}$ Institute for Theoretical and Experimental Physics, Moscow, Russia

${ }^{24}$ Lebedev Physical Institute, Moscow, Russia ${ }^{e, h}$

${ }^{25}$ Max-Planck-Institut für Physik, München, Germany ${ }^{a}$

${ }^{26}$ LAL, Université de Paris-Sud, IN2P3-CNRS, Orsay, France

${ }^{27}$ LPNHE, Ecole Polytechnique, IN2P3-CNRS, Palaiseau, France

${ }^{28}$ LPNHE, Universités Paris VI and VII, IN2P3-CNRS, Paris, France

${ }^{29}$ Institute of Physics, Czech Academy of Sciences, Praha, Czech Republic ${ }^{e, i}$

${ }^{30}$ Faculty of Mathematics and Physics, Charles University, Praha, Czech Republic ${ }^{e, i}$

${ }^{31}$ Dipartimento di Fisica Università di Roma Tre and INFN Roma 3, Roma, Italy

32 Paul Scherrer Institut, Villigen, Switzerland 
${ }^{33}$ Fachbereich Physik, Bergische Universität Gesamthochschule Wuppertal, Wuppertal, Germany $^{a}$

${ }^{34}$ Yerevan Physics Institute, Yerevan, Armenia

${ }^{35}$ DESY, Zeuthen, Germany ${ }^{a}$

${ }^{36}$ Institut für Teilchenphysik, ETH, Zürich, Switzerland ${ }^{j}$

${ }^{37}$ Physik-Institut der Universität Zürich, Zürich, Switzerland ${ }^{j}$

${ }^{38}$ Also at Physics Department, National Technical University, Zografou Campus, GR-15773 Athens, Greece

${ }^{39}$ Also at Rechenzentrum, Bergische Universität Gesamthochschule Wuppertal, Germany

${ }^{40}$ Also at Institut für Experimentelle Kernphysik, Universität Karlsruhe, Karlsruhe, Germany

${ }^{41}$ Also at Dept. Fis. Ap. CINVESTAV, Mérida, Yucatán, México ${ }^{k}$

${ }^{42}$ Also at University of P.J. Šafárik, Košice, Slovak Republic

${ }^{43}$ Also at CERN, Geneva, Switzerland

$\dagger$ Deceased

${ }^{a}$ Supported by the Bundesministerium für Bildung, Wissenschaft, Forschung und Technologie, FRG, under contract numbers 7AC17P, 7AC47P, 7DO55P, 7HH17I, 7HH27P, 7HD17P, 7HD27P, 7KI17I, 6MP17I and 7WT87P

${ }^{b}$ Supported by the UK Particle Physics and Astronomy Research Council, and formerly by the UK Science and Engineering Research Council

${ }^{c}$ Supported by FNRS-NFWO, IISN-IIKW

${ }^{d}$ Partially Supported by the Polish State Committee for Scientific Research, grant no. 2P0310318 and SPUB/DESY/P03/DZ-1/99, and by the German Federal Ministry of Education and Science, Research and Technology (BMBF)

e Supported by the Deutsche Forschungsgemeinschaft

${ }^{f}$ Supported by VEGA SR grant no. 2/5167/98

${ }^{g}$ Supported by the Swedish Natural Science Research Council

${ }^{h}$ Supported by Russian Foundation for Basic Researc grant no. 96-02-00019

${ }^{i}$ Supported by GA AV ČR grant no. A1010821

${ }^{j}$ Supported by the Swiss National Science Foundation

${ }^{k}$ Supported by CONACyT 


\section{Introduction}

Deep-inelastic lepton-proton scattering (DIS) experiments have played a fundamental role in establishing Quantum-Chromodynamics (QCD) as the theory of the strong interaction and in the understanding of the structure of the proton. The lepton inclusive DIS cross section is directly sensitive to the quark densities in the proton, but gives only indirect information on the gluon content and on the strong coupling constant $\alpha_{s}$ via scaling violations of the structure functions. The production rates of events in which the final state contains more than one hard jet (besides the proton remnant) are, however, observables which are directly sensitive to both $\alpha_{s}$ and the gluon density in the proton. These multi-jet cross sections can thus be used to test the predictions of perturbative QCD (pQCD) and allow a direct determination of $\alpha_{s}$ and the gluon density [1].

The large center-of-mass energy $\sqrt{s}$ of $300 \mathrm{GeV}$ at HERA allows multi-jet production in DIS to be studied over large regions of phase space. In this paper we present comprehensive measurements of jet production in the range of four-momentum transfers squared $5<Q^{2}<$ $15000 \mathrm{GeV}^{2}$. Using four different jet algorithms we study multi-differential distributions of the dijet and the inclusive jet cross sections to which the predictions of pQCD in next-to-leading order in $\alpha_{s}$ are compared. We identify those observables for which theoretical predictions have small uncertainties and perform QCD analyses of the jet data in which we determine the value of $\alpha_{s}$. A consistent determination of the gluon density in the proton, together with the quark densities is obtained in a simultaneous fit which additionally includes data on the inclusive DIS cross section. In the last step an analysis of the data in which both $\alpha_{s}$ and the gluon density are determined simultaneously is presented.

This paper is organized as follows. In section 2 we give a short description of the theoretical framework and motivate the choice of the jet variables to be measured. The experimental environment and details of the measurement procedure are described in section 3 and the multidifferential jet cross sections are presented in section 4. Finally, in section 5 we introduce the theoretical assumptions and the methods which are used in the QCD analysis and present the results of the QCD fits. Numerical values of the results are given as tables in the appendix.

\section{Jet Production in Deep-Inelastic Scattering}

\subsection{Jet Variables and the Breit Frame}

The inclusive neutral current cross section in deep-inelastic lepton-proton scattering is described in lowest order perturbation theory as the scattering of the lepton off a quark in the proton via the exchange of a virtual gauge boson $\left(\gamma, Z^{0}\right)$ (according to Fig. 1 (a)). The kinematics of the reaction are given by the four-momentum transfer squared $Q^{2}$, the Bjorken scaling variable $x_{\mathrm{Bj}}$ and the inelasticity $y$ defined as

$$
Q^{2} \equiv-q^{2}=-\left(l-l^{\prime}\right)^{2} \quad x_{\mathrm{Bj}} \equiv \frac{Q^{2}}{2 p \cdot q} \quad y \equiv \frac{p \cdot q}{p \cdot l}
$$




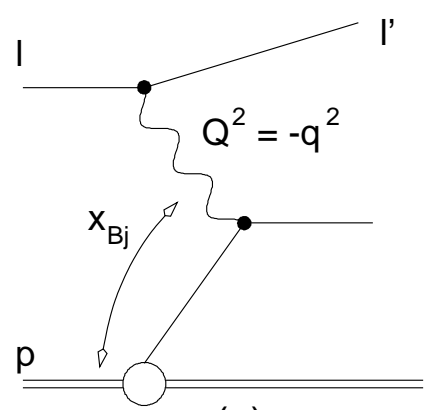

(a)

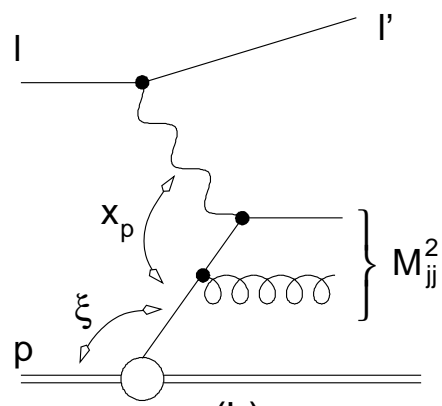

(b)

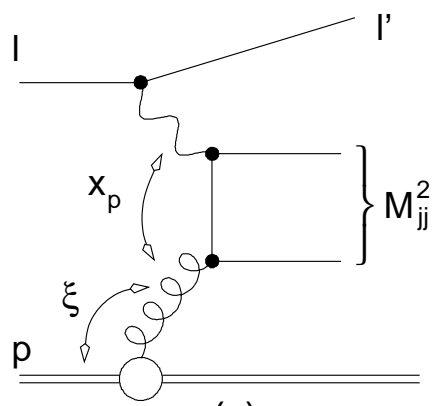

(c)

Figure 1: Diagrams of different processes in deep-inelastic lepton-proton scattering: (a) Born process , (b) QCD-Compton process and (c) the boson-gluon fusion.

where $l\left(l^{\prime}\right)$ and $p$ are the four-momenta of the initial (final) state lepton and proton, respectively. When particle masses are neglected the kinematic variables are related to the lepton-proton center-of-mass energy $\sqrt{s}$ by $s x_{\mathrm{Bj}} y=Q^{2}$. The variable $x_{\mathrm{Bj}}$ is in the leading order approximation identical with the longitudinal momentum fraction $x$ of the proton which is carried by the parton specified by the parton density functions, hereafter referred to as the struck parton!.

Multi-jet production in DIS is described by the QCD-Compton and the boson-gluon fusion processes. Due to the latter contribution multi-jet cross sections are directly sensitive to the gluon density in the proton. Examples of leading order diagrams of both processes are shown in Fig. 1 (b) and (c). Both diagrams contribute to the cross section which depends explicitly on $\alpha_{s}$. Variables that characterize features of the multi-jet final state are the invariant mass $M_{\mathrm{jj}}$, the partonic scaling variable $x_{p}$ and the variable $\xi$ defined as

$$
\xi \equiv x_{\mathrm{Bj}}\left(1+\frac{M_{\mathrm{jj}}^{2}}{Q^{2}}\right) \quad \text { and } \quad x_{p} \equiv \frac{x_{\mathrm{Bj}}}{\xi}
$$

In the leading order picture (when the final state partons are identified with jets) the invariant dijet mass $M_{\mathrm{jj}}$ is equal to the center-of-mass energy of the boson-parton reaction. In this approximation the fractional momentum $x$ of the struck parton is given by the variable $\xi$ which becomes much larger than the Bjorken scaling variable $x_{\mathrm{Bj}}$ if $M_{\mathrm{jj}}$ is large. The partonic scaling variable $x_{p}$ specifies the fractional momentum of the incoming parton seen by the boson.

Studies of the dynamics of multi-jet production are preferably performed in the Breit frame where the virtual boson interacts head-on with the proton [2]. The Breit frame is defined by $2 x_{\mathrm{Bj}} \vec{p}+\vec{q}=0$, where $\vec{p}$ and $\vec{q}$ are the momenta of the proton and the exchanged boson, respectively. The positive $z$-axis is chosen to be the proton direction. In the lowest order process, at $\mathcal{O}\left(\alpha_{s}^{0}\right)$, the quark from the proton is back-scattered into the negative $z$-direction and no transverse energy is produced [? The appearance of jets with large transverse energies $E_{T}$ can only be explained by hard QCD processes whose contribution is at least of $\mathcal{O}\left(\alpha_{s}\right)$ relative to the

\footnotetext{
${ }^{1}$ In this paper the Bjorken scaling variable $x_{\mathrm{Bj}}$ is always written with a subscript to distinguish it from the proton momentum fraction $x$ which appears in the formulae of the proton's parton densities. While the former is an observable quantity, the latter is only defined in a theoretical framework within a given factorization scheme.

${ }^{2}$ By "transverse" we refer to the component perpendicular to the $z$-axis. The transverse energy is defined as $E_{T} \equiv E \sin \theta$. The polar angle $\theta$ is defined with respect to the proton direction in both the laboratory frame and the Breit frame. Throughout the paper "transverse energy" always refers to transverse energies in the Breit frame.
} 
gluon-photon center-of-mass frame

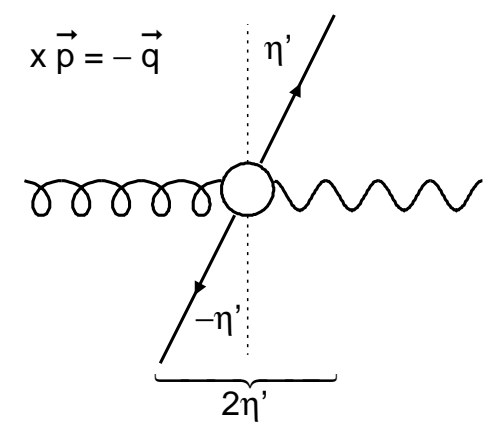

Breit frame

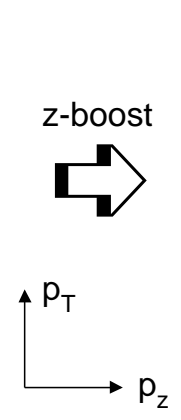

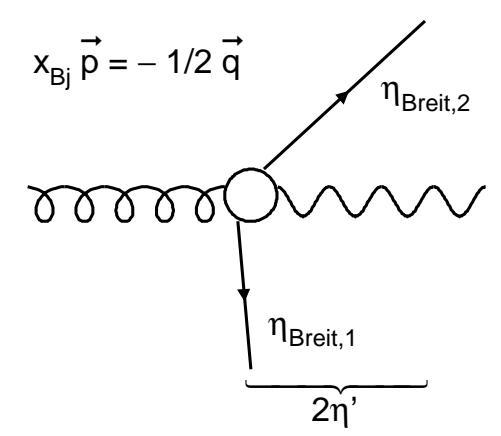

Figure 2: A boson-gluon fusion event in deep-inelastic scattering in the boson-gluon centerof-mass frame (left) and in the Breit frame (right). The frames are related to each other by a longitudinal boost along the $z$-direction.

inclusive DIS cross section. The hardness of the QCD process is specified by $E_{T}$ which is the physical scale at which e.g. hard gluon radiation is resolved.

In the leading order approximation the Breit frame is related to the boson-parton rest frame by a longitudinal boost along the $z$-direction (see Fig. 2). The polar scattering angle of the jets in the boson-parton center-of-mass frame is directly related to the pseudorapidity $\eta^{\prime}$ of the jets. In leading order approximation the value of $\eta^{\prime}$ is equal to half the difference of the jet pseudorapidities $\eta_{\mathrm{Breit}}$ in the Breit frame $\eta^{\prime}=\frac{1}{2}\left|\eta_{\mathrm{Breit}, 1}-\eta_{\mathrm{Breit}, 2}\right|$. Since the transverse jet energy $E_{T}$ is invariant under longitudinal boosts along the $z$-axis $E_{T}$ is identical in both frames.

\subsection{Jet Definitions}

The comparison of the properties of high multiplicity hadronic final states observed in the experiment to those in perturbative calculations involving only a small number of partons requires the definition of infrared- and collinear-safe jet observables. While the properties of different jet observables depend on the exact definition of the jets the physical interpretation of experimental results must, however, not depend on details of the jet definition if theory is to be claimed successful.

In this analysis we use four jet clustering algorithms which successively recombine particles into jets. All jet algorithms are applied in the Breit frame to the final state particlesff excluding the scattered lepton. They can be grouped into two pairs of inclusive and exclusive jet algorithms, each pair consisting of one $k_{\perp}$ ordered and one angular ordered algorithm. In the $k_{\perp}$ (angular) ordered algorithms pairs of particles are clustered in the order of increasing relative transverse momenta $k_{\perp}$ (increasing angles) between the particles. The exclusive jet definitions assign each particle explicitly to a hard jet or to the proton remnant, while for the inclusive

\footnotetext{
${ }^{3}$ The pseudorapidity is defined as $\eta \equiv-\ln (\tan \theta / 2)$ where $\theta$ is the polar angle. Positive values of $\eta$ correspond to particle momenta pointing into the proton hemisphere. For massless particles, differences in pseudorapidity are invariant under longitudinal boosts.

4"Particle" refers in this paper either to an energy deposit or a track in the detector, to a parton in a perturbative calculation or to a hadron (i.e. any particle produced in the hadronization process including soft photons and leptons from secondary decays). All particles are treated as massless by a redefinition of the energy $(E \equiv|\vec{p}|)$.
} 
jet definitions not all particles are necessarily assigned to hard jets. The following four jet algorithms are used:

- the exclusive $k_{\perp}$ ordered algorithm as proposed in [3].

- the exclusive angular ordered algorithm (Cambridge algorithm) as proposed in [ 4 ] and modified for DIS to consider the proton remnant as a particle of infinite momentum along the positive $z$-axis, following the approach used in [3]. The exact definition is taken from [5].

- the inclusive $k_{\perp}$ ordered algorithm as proposed in [6, 7].

- the inclusive angular ordered algorithm (Aachen algorithm) as proposed in [5, 8]. In analogy to the changes from the exclusive $k_{\perp}$ algorithm to the Cambridge algorithm, the inclusive $k_{\perp}$ algorithm has been modified to obtain an inclusive jet algorithm with angular ordering.

The recombination of particles in the exclusive jet algorithms is made in the $E$-scheme (addition of four-vectors) resulting in massive jets. To maintain invariance under longitudinal boosts for the inclusive jet definitions the $E_{T}$ recombination scheme [9] is used in which the resulting jets are massless.

In the exclusive jet definitions the clustering procedure is stopped when the distances $y_{i j}=$ $k_{\perp i j}^{2} / S^{2}$ defined between all pairs of jets and between all jets and the proton remnant are above some value $y_{\text {cut }}$, where $k_{\perp i j}^{2}=2 \min \left(E_{i}^{2}, E_{j}^{2}\right)\left(1-\cos \theta_{i j}\right)$ and $S$ is a reference scale. In our analysis we set $S^{2}=100 \mathrm{GeV}^{2}$ and $y_{\text {cut }}=1$ to have the final jets separated by $k_{\perp i j}>10 \mathrm{GeV}$. The inclusive jet algorithms are independent of an explicit stopping criterion in the clustering procedure and hard jet selection cuts have to be applied afterwards. These algorithms are defined by a radius parameter $R_{0}$ which we set to $R_{0}=1$ as suggested in [6].

\subsection{Phase Space}

The kinematic region in which the analysis is performed is defined by the kinematic variables $y$ and $Q^{2}$

$$
0.2<y<0.6 \quad \text { and } \quad 5<Q^{2}<15000 \mathrm{GeV}^{2}
$$

The lower limit on $y$ has been chosen to exclude the kinematic region of large $x_{\mathrm{Bj}}$ where jets are predominantly produced in the forward direction, i.e. at the edge of the detector acceptance. The upper limit on $y$ ensures large energies of the scattered lepton.

The jet finding is performed using the jet algorithms introduced above. We restrict the jet phase space to the angular range in which jets can be well measured in the H1 detector. Therefore the four-vectors of the jets defined in the Breit frame are boosted to the laboratory frame where we then apply the pseudorapidity cut

$$
-1<\eta_{\text {jet, lab }}<2.5 \text {. }
$$


In this kinematic range double-differential jet cross sections are measured as a function of $Q^{2}$, $E_{T}$ (inclusive jet cross section) and $\bar{E}_{T}=\frac{1}{2}\left(E_{T 1}+E_{T 2}\right)$ (dijet cross section) using the various jet algorithms mentioned above. In addition the dependences of the dijet cross section on the dijet variables $M_{\mathrm{jj}}, \xi, x_{p}$ and $\eta^{\prime}$ as introduced in section 2.1 as well as on the pseudorapidity of the forward jet $\eta_{\text {forw, lab }}$ in the laboratory frame are measured. In all cases inclusive dijet cross sections, i.e. cross sections to produce two or more jets within the angular acceptance are measured. The jet variables are calculated from the two jets with highest transverse energy (the jets are labeled in the order of descending $E_{T}$ ).

In the measurement of the dijet cross section care has to be taken to avoid regions at the boundary of phase space which are sensitive to soft gluon emissions where perturbative calculations in fixed order are not able to make reliable predictions. The exclusive jet algorithms avoid these regions due to the cut on the variable $k_{\perp}$. For the inclusive jet definitions additional selection cuts have to be chosen appropriately. The dijet cross section defined by a symmetric cut on the transverse energy of the jets $E_{T, 1,2}>E_{\mathrm{T}, \min }$ is infrared sensitive [10]. This problem can be avoided by an additional, substantially harder cut on, for example either a) the sum $E_{T, 1}+E_{T, 2}$, b) $E_{T, 1}$ or c) the invariant dijet mass $M_{\mathrm{jj}}$. When cuts are chosen to obtain cross sections of similar size in all of a), b) and c) above, the next-to-leading order corrections are largest in b) and hadronization corrections are largest in c). The smallest next-to-leading order corrections and hadronization effects are seen for scenario a). For the inclusive jet algorithms we therefore require

$$
E_{T, 1,2}>5 \mathrm{GeV} \quad \text { and } \quad E_{T, 1}+E_{T, 2}>17 \mathrm{GeV} .
$$

\subsection{QCD Predictions of Jet Cross Sections}

While leading order (LO) calculations can predict the order of magnitude and the rough features of an observable, reliable quantitative predictions require the perturbative calculations to be performed (at least) to next-to-leading order (NLO). The NLO calculations of the jet cross sections used in this analysis are performed in the $\overline{\mathrm{MS}}$ scheme for five massless quark flavors using the program DISENT [11] which has been tested in [12] and found to agree with the program DISASTER++ [13] in the kinematic region of interest.

Perturbative fixed order calculations beyond leading order can give reliable quantitative predictions for observables with small sensitivity to multiple emission effects and non-perturbative contributions. They fail, however, to predict details of the structure of multi-particle final states as observed in the experiment. A complementary approach to describe these properties of the hadronic final state is used in parton cascade models. Starting from the leading order matrix elements, subsequent emissions are calculated based on soft and collinear approximations. There exist two different approaches in which parton emissions are either described by a parton shower model (HERWIG [14], LEPTO [15] and RAPGAP [16]) or by a dipole cascade (ARIADNE [17]). These parton cascade models can be matched to phenomenological models of the hadronization process. The HERWIG event generator uses the cluster fragmentation model [18] while in LEPTO, RAPGAP and ARIADNE the Lund string model [19] is implemented. The programs HERWIG, LEPTO, RAPGAP and ARIADNE are used in the present measurement

\footnotetext{
${ }^{5}$ We have modified DISENT to include the running of the electromagnetic coupling constant.
} 

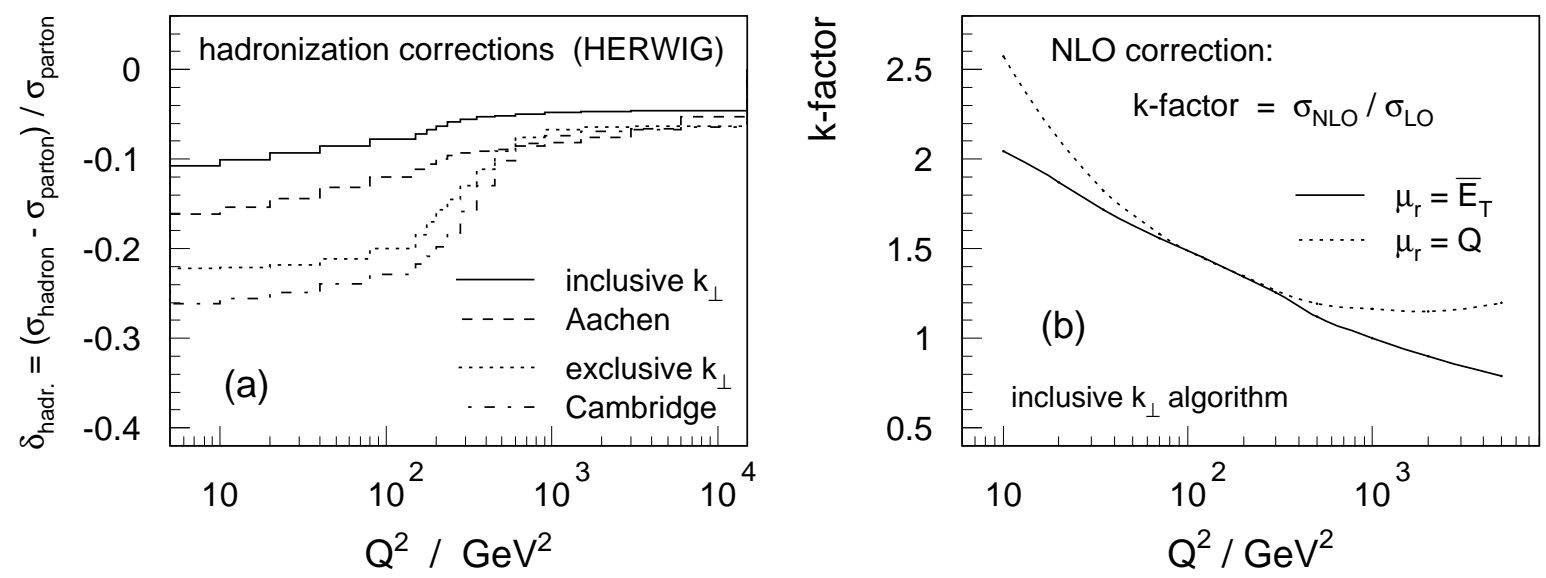

Figure 3: The predictions of (a) the hadronization corrections to the dijet cross section for different jet definitions as a function of $Q^{2}$ as obtained by HERWIG and (b) the next-to-leading order corrections to the dijet cross section as a function of $Q^{2}$ for the inclusive $k_{\perp}$ algorithm using two different renormalization scales $\mu_{r}$.

to provide event samples which are used in the correction procedure for the data. In the QCD analysis they are used to estimate the size of the hadronization corrections to the perturbative jet cross sections.

Higher order QED corrections can change the size of the cross section and also modify the event topology. Especially hard photon radiation may strongly influence the reconstruction of the event kinematics and thereby the boost vector to the Breit frame. Corrections from real photon emissions from the lepton and virtual corrections at the leptonic vertex are included in the program HERACLES [20] which is directly interfaced to RAPGAP. An interface to LEPTO and ARIADNE is provided by the program DJANGO [21].

Safe predictions can only be expected for observables for which perturbative higher-order corrections and non-perturbative (hadronization) corrections are small. Detailed investigations on properties of the NLO cross sections and the size and the uncertainties of the hadronization corrections to the observables under study have been performed in [5, 8, 22]. The hadronization corrections predicted from the different models are in good agreement and have small sensitivity to model parameters. The hadronization corrections $\delta_{\text {hadr. }}$ are displayed in Fig. 3 (a) as a function of $Q^{2}$ for all jet algorithms used. They are defined as $\delta_{\text {hadr. }}=\left(\sigma_{\text {hadron }}-\sigma_{\text {parton }}\right) / \sigma_{\text {parton }}$ where $\sigma_{\text {parton }}\left(\sigma_{\text {hadron }}\right)$ is the jet cross section before (after) hadronization. The hadronization corrections are generally smaller for the inclusive jet algorithms than for the exclusive ones and smaller for the $k_{\perp}$ ordered algorithms when compared to those with angular ordering. Hence the inclusive $k_{\perp}$ algorithm shows the smallest corrections, acceptable even down to very low $Q^{2}$ values. Having the smallest hadronization corrections, the inclusive $k_{\perp}$ algorithm is thus the best choice for a jet definition. The other jet algorithms will, however, still be used to demonstrate the consistency of the results.

An indication of the possible size of perturbative higher-order contributions is given by the size of the NLO corrections or the renormalization and factorization scale dependence of an observable. For the inclusive $k_{\perp}$ algorithm the NLO corrections to the dijet cross section are displayed in Fig. 3 (b). Shown is the $k$-factor, defined as the ratio of the NLO and the LO 
predictions, for two different choices of the renormalization scale $\left(\mu_{r}=\bar{E}_{T}, Q\right)$. Towards low $Q^{2}$ the NLO corrections become large, especially for the choice $\mu_{r}=Q$. Reasonably small $k$ factors $(k<1.4)$ are only seen at $Q^{2} \gtrsim 150 \mathrm{GeV}^{2}$ where $Q^{2}$ and $E_{T}^{2}$ are of similar size such that terms $\propto \ln \left(E_{T}^{2} / Q^{2}\right)$ are small. The renormalization scale dependence is seen to be correlated with the NLO correction i.e. large at small $Q^{2}$. The factorization scale dependence is below $2 \%$ over the whole phase space (not shown). These studies suggest that a QCD analysis of jet cross sections, involving the determination of $\alpha_{s}$ and the gluon density, should be performed at large values of $Q^{2}$.

\section{Experimental Technique}

The analysis is based on data taken in the years 1995-1997 with the H1 detector at HERA in which positrons with energies of $E_{e}=27.5 \mathrm{GeV}$ collided with protons with energies of $E_{p}=820 \mathrm{GeV}$.

\subsection{H1 Detector}

A detailed description of the $\mathrm{H} 1$ detector can be found elsewhere [23]. Here we briefly introduce the detector components most relevant for this analysis.

In the polar angular range $4^{\circ}<\theta<154^{\circ}$ the electromagnetic and hadronic energy is measured by the Liquid Argon (LAr) calorimeter [24] with full azimuthal coverage. The LAr calorimeter consists of an electromagnetic section (20-30 radiation lengths) with lead absorbers and a hadronic section with steel absorbers. The total depth of both sections varies between 4.5 and 8 interaction lengths. Test beam measurements of the LAr calorimeter modules have shown an energy resolution of $\sigma_{E} / E \approx 0.12 / \sqrt{E[\mathrm{GeV}]} \oplus 0.01$ for electrons [25] and $\sigma_{E} / E \approx$ $0.50 / \sqrt{E[\mathrm{GeV}]} \oplus 0.02$ for charged pions after software weighting [26].

In the backward direction $\left(153^{\circ}<\theta<177^{\circ}\right)$ energy is detected by a lead-fiber calorimeter, SPACAL [27]. It consists of an electromagnetic section with a depth of 28 radiation lengths in which the scattered positron is measured with an energy resolution of $\sigma_{E} / E=$ $0.071 / \sqrt{E[\mathrm{GeV}]} \oplus 0.010$. It is complemented by a hadronic section to yield a total depth of two interaction length.

Charged particle tracks are measured in two concentric jet drift chamber modules (CJC), covering the polar angular range $25^{\circ}<\theta<165^{\circ}$. A forward tracking detector covers $7^{\circ}<$ $\theta<25^{\circ}$ and consists of drift chambers with alternating planes of parallel wires and others with wires in the radial direction. A backward drift chamber BDC improves the identification of the scattered positron in the SPACAL calorimeter. The calorimeters and the tracking chambers are surrounded by a superconducting solenoid providing a uniform magnetic field of $1.15 \mathrm{~T}$ parallel to the beam axis in the tracking region.

The luminosity is measured using the Bethe-Heitler process $e p \rightarrow e \gamma p$. The final state positron and photon are detected in calorimeters situated close to the beam pipe at distances of $33 \mathrm{~m}$ and $103 \mathrm{~m}$ from the interaction point in the positron beam direction. 


\subsection{Event Selection}

Neutral current DIS events are triggered and identified by the detection of the scattered positron as a compact electromagnetic cluster. The data set is divided into two subsamples in which the positron is detected either in the SPACAL $\left(5<Q^{2}<70 \mathrm{GeV}^{2}\right)$ or in the LAr calorimeter $\left(150<Q^{2}<15000 \mathrm{GeV}^{2}\right)$ with uniform acceptance over the range $0.2<y<0.6$. These regions are labeled "low $Q^{2}$ " and "high $Q^{2}$ " throughout the text. The low $Q^{2}$ and high $Q^{2}$ samples correspond to integrated luminosities of $\mathcal{L}_{\text {int }} \simeq 21 \mathrm{pb}^{-1}$ and $\mathcal{L}_{\text {int }} \simeq 33 \mathrm{pb}^{-1}$, respectively!.

At low $Q^{2}$ the positron is reconstructed as the highest electromagnetic energy cluster in the SPACAL, requiring an energy of $E_{e}^{\prime}>10 \mathrm{GeV}$ and a polar angle of $156^{\circ}<\theta_{\mathrm{e}}<176^{\circ}$. The positron selection at high $Q^{2}$ closely follows the procedure used in the recent measurement of the inclusive DIS cross section [28], requiring an electromagnetic cluster of $E_{e}^{\prime}>12 \mathrm{GeV}$ with a polar angle $\theta_{\mathrm{e}} \lesssim 153^{\circ}$. For $\theta_{e}>35^{\circ}$ the positron candidate is validated only if it can be associated with a reconstructed track, which points to the positron cluster. Fiducial cuts are applied to avoid the boundary regions between the calorimeter modules in the $z$ and $\phi$ (i.e. azimuthal) directions. The events in the low and high $Q^{2}$ samples are triggered by demanding a localized energy deposition together with loose track requirements. The trigger efficiencies for the final jet event samples are above $98 \%$.

In both samples the reconstructed $z$-coordinate of the event vertex is required to be within $\pm 35 \mathrm{~cm}$ of its nominal position. The hadronic final state is reconstructed from a combination of low momentum tracks $\left(p_{T}<2 \mathrm{GeV}\right)$ in the central jet chamber and energy deposits measured in the LAr calorimeter and in the SPACAL according to the prescription in [28]. From momentum conservation the sum $\sum\left(E-p_{z}\right)$ over all hadronic final state particles and the scattered positron is expected to be $2 E_{e}=55 \mathrm{GeV}$. This value is lowered in events in which particles escape undetected in the beam pipe in negative $z$-direction. Photoproduction background and events with hard photon radiation collinear to the positron beam are therefore suppressed by a cut on $45<\sum\left(E-p_{z}\right)<65 \mathrm{GeV}$.

The event kinematics is determined from a redundant set of variables using the scattered positron and the hadronic final state. Using all hadronic final state particles $h$ the variable $\sum=\sum_{h}\left(E_{h}-p_{z, h}\right)$ is derived. The kinematic variables $x_{\mathrm{Bj}}, y$ and $Q^{2}$ are then reconstructed by the Electron-Sigma method [29]

$$
\begin{gathered}
Q_{e \Sigma}^{2}=4 E_{e} E_{e}^{\prime} \cos ^{2} \frac{\theta_{e}}{2} \quad x_{e \Sigma}=\frac{E_{e}^{\prime 2} \sin ^{2} \theta_{e}}{s y_{\Sigma}\left(1-y_{\Sigma}\right)} \quad y_{e \Sigma}=\frac{2 E_{e}}{\Sigma+E_{e}^{\prime}\left(1-\cos \theta_{e}\right)} y_{\Sigma} \\
\text { with } \quad y_{\Sigma}=\frac{\Sigma}{\Sigma+E_{e}^{\prime}\left(1-\cos \theta_{e}\right)} .
\end{gathered}
$$

The jet algorithms are applied to the hadronic final state particles which are boosted to the Breit frame. The boost vector is determined from the variables $y_{e \Sigma}, Q_{e \Sigma}^{2}$ and the azimuthal angle of the scattered positron. The transverse jet energy $E_{T}$ (or $E_{T}$ ), the dijet mass $M_{\mathrm{jj}}$ and the variable $\eta^{\prime}$ are calculated from the four-vectors of the jets. The variables $\xi$ and $x_{p}$ are reconstructed as

$$
\xi_{\text {rec }}=x_{\mathrm{e} \Sigma}+\frac{M_{\mathrm{jj}}^{2}}{y_{\mathrm{h}} s} \quad \text { and } \quad x_{p, \text { rec }}=\frac{x_{e \Sigma}}{\xi_{\text {rec }}} \quad \text { with } \quad y_{\mathrm{h}}=\frac{\Sigma}{2 E_{e}} .
$$

\footnotetext{
${ }^{6}$ The low $Q^{2}$ sample uses only data from the years 1996-1997.
} 
These relations exploit partial cancellations in the hadronic energy measurement in $M_{\mathrm{jj}}^{2}$ and $y_{\mathrm{h}}$.

The fraction of dijet events in the inclusive neutral current DIS event sample varies strongly with $Q^{2}$, namely between $\simeq 1 \%$ (at $Q^{2}=5 \mathrm{GeV}^{2}$ ) and $\simeq 20 \%$ (at $Q^{2}=5000 \mathrm{GeV}^{2}$ ). Using the inclusive $k_{\perp}$ algorithm we have selected 11400 dijet events at low $Q^{2}$ and 2855 dijet events at high $Q^{2}$. The inclusive jet sample (measured only at high $Q^{2}$ ) contains 10432 jets with $E_{T}>7 \mathrm{GeV}$, from 7263 events. The size of photoproduction background has been estimated using two samples of photoproduction events generated by PYTHIA [30] and PHOJET [31]. The contribution to the distributions of the finally selected events is found to be negligible (i.e. below $1 \%$ ) in all variables under study.

\subsection{Correction Procedure}

The data are corrected for effects of limited detector resolution and acceptance, as well as for inefficiencies of the selection and higher order QED corrections. The latter are dominated by real photon emissions from the positron (initial- and final-state radiation) and virtual corrections at the leptonic vertex, as included in the program HERACLES. No further corrections for effects due to the running of the electromagnetic coupling constant or non-perturbative processes (i.e. hadronization) are applied.

To determine the correction functions the generators LEPTO, RAPGAP and ARIADNE (all interfaced to HERACLES) are used. For each generator two event samples are generated. The first sample, which includes QED corrections, is subjected to a detailed simulation of the H1 detector based on GEANT [32]. The second event sample is generated under the same physics assumptions, but without QED corrections and without detector simulation. The correction functions are determined bin-wise for each observable as the ratio of its value in the second sample and its value in the first sample. This method can be used if migrations between different bins are small and properties of the simulated events are similar to those of the data. The absolute normalization of the generated cross sections is, however, arbitrary, since this cancels in the ratio.

To test their applicability for the correction procedure, detailed comparisons have been made of the simulated event samples and the data for a multitude of jet distributions [5]. None of the models can describe the magnitude of the jet cross section; especially at low $Q^{2}$ large deviations are seen. However, all models give a reasonable description of the properties of the hadronic final state and of the properties of single jets and the dijet system including angular jet distributions. In a previous publication [33] it has been shown that these event generators give a good description of the internal structure of jets.

Based on the event simulation the bin sizes of the observables are chosen to match the resolution. The final bin purities and efficiencies are typically above 50\% and migrations are sufficiently small to have small correlations between adjacent bins. The correction functions as determined by different event generators are in good agreement with each other and the absolute values deviate typically by less than $20 \%$ from unity. The final correction functions applied to the data are taken to be the average values from the different models. The difference between the average and the single values are quoted as the uncertainty induced by the model dependence which is subdivided in equal fractions into correlated and uncorrelated uncertainty between data points. This uncertainty is typically below $4 \%$. 


\subsection{Experimental Uncertainties}

In addition to the model dependence of the correction function and the statistical uncertainties of the data and of the correction function several other sources of systematic experimental uncertainties are studied. They are given in the following, together with the typical change of the cross sections and a remark whether a particular uncertainty is treated as correlated or uncorrelated between different data points. The latter classification closely follows the one used in [28].

- The measurement of the integrated luminosity introduces an overall normalization uncertainty of $\pm 1.5 \%$; correlated.

- The hadronic energy scale of the LAr calorimeter is varied by $\pm 4 \%$; $\pm 2 \%$ of the effect is considered to be correlated; typical change of the cross sections $\pm 7.5 \%$.

- The hadronic energy scale of the SPACAL is varied by $\pm 7 \%$;

typical change of the cross sections $< \pm 1 \%$; uncorrelated.

- The track momenta of the hadronic final state are varied by $\pm 3 \%$; typical change of the cross sections $\pm 2.5 \%$; uncorrelated.

- The calibration of the positron energy in the SPACAL is varied by $\pm 1 \%$; typical change of the cross sections $< \pm 2 \%$; correlated.

- The positron calibration of the LAr calorimeter is treated as in [28]; a variation between $\pm 0.7 \%$ and $\pm 3 \%$ is made, depending on the $z$-position of the energy cluster in the detector, from which $\pm 0.5 \%$ is considered to be correlated between different data points; the rest is treated as uncorrelated; typical change of the cross sections $\pm 4 \%$.

- The positron polar angle is varied by $\pm 2 \mathrm{mrad}( \pm 3 \mathrm{mrad}$ ) for positrons in the SPACAL (LAr calorimeter); typical change of the cross sections $< \pm 2 \%$; correlated.

- The positron azimuthal angle is varied by $\pm 3 \mathrm{mrad}$;

typical change of the cross sections $< \pm 1 \%$; uncorrelated.

The largest experimental uncertainty comes from the uncertainty of the energy scale of the LAr calorimeter. Since the uncertainties from all other sources are fairly small their determination is often subject to fluctuations. We therefore give a conservative estimate, by quoting the maximal (up- and downward) variation as the symmetric uncertainty.

The statistical and the uncorrelated systematic uncertainties are added in quadrature to obtain the total uncorrelated uncertainty. The correlated contributions are kept separately and can thus be considered in a statistical analysis. To obtain the total uncertainty for each single data point, all contributions are added in quadrature. 

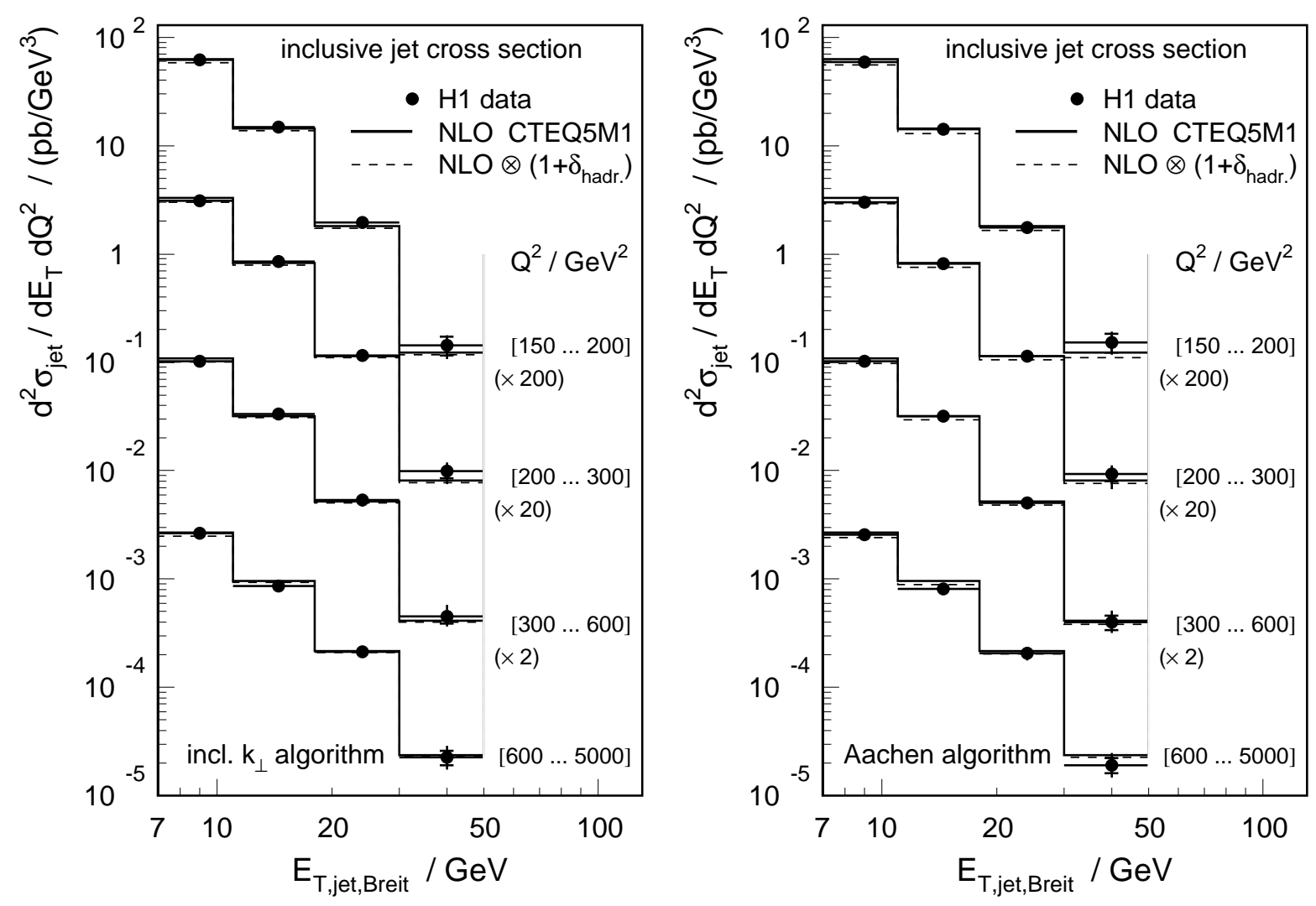

Figure 4: The inclusive jet cross section as a function of the transverse jet energy in different regions of $Q^{2}$ for the inclusive $k_{\perp}$ algorithm (left) and for the Aachen algorithm (right). The data are compared to the perturbative QCD prediction in NLO with (dashed line) and without (solid line) hadronization corrections included.

\section{Experimental Results}

The measured cross sections, corrected for detector effects and effects of higher order QED, are presented as single- or double-differential distributions where the inner (outer) error bars represent the statistical (total) uncertainty of the data points. The results (defined in the phase space specified in section 2.3) are directly compared to the perturbative QCD predictions in NLO. The hadronization corrections $\delta_{\text {hadr. }}$ have been estimated using the models described in section 2.4 for which the predictions are in good agreement with each other. In all the figures shown the theoretical prediction "NLO $\otimes\left(1+\delta_{\text {hadr. }}\right)$ " is derived from the NLO calculations with hadronization corrections determined using HERWIG. All NLO calculations are performed using the parton density parameterizations CTEQ5M1 [34] and a value of $\alpha_{s}\left(M_{Z}\right)=0.118$. The renormalization scale is set to the transverse jet energy $\mu_{r}=E_{T}$ or in case of the dijet cross section to the average transverse energy $\bar{E}_{T}$. For the factorization scale a fixed value' of $\mu_{f}=\sqrt{200} \mathrm{GeV}$, corresponding to the average $E_{T}$ of the jet sample, is chosen.

\footnotetext{
${ }^{7}$ This slightly unusual procedure is motivated in section 5.2. A variation of $\mu_{f}$ in the range $6<\mu_{f}<30 \mathrm{GeV}$ changes the NLO results by less than $2 \%$.
} 


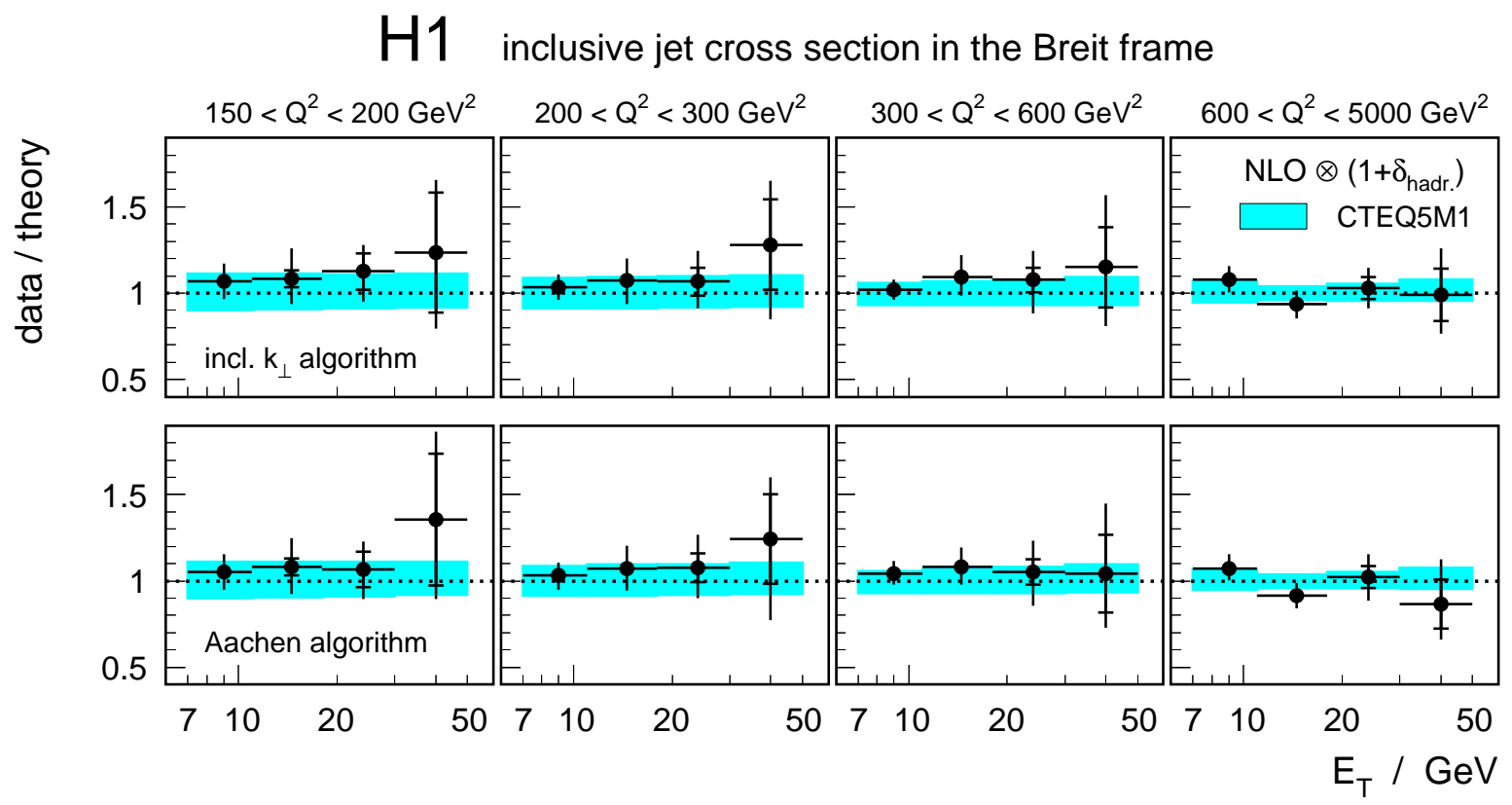

Figure 5: The ratio of the measured inclusive jet cross section and the theoretical prediction for the inclusive $k_{\perp}$ algorithm (top) and the Aachen algorithm (bottom). The uncertainty of the theoretical prediction is indicated by the band (the contributions from the renormalization and factorization scale dependence and the hadronization corrections are added in quadrature).

\subsection{Inclusive Jet Cross Section}

The inclusive jet cross section is measured at high $Q^{2}$ in the Breit frame for both inclusive jet algorithms. The results are presented in Fig. 7 double-differentially as a function of the transverse jet energy in the Breit frame $E_{T}$ in different regions of $Q^{2}$. The data for the inclusive $k_{\perp}$ algorithm (left) and for the Aachen algorithm (right) cover a range of transverse jet energies squared $\left(49<E_{T}^{2}<2500 \mathrm{GeV}^{2}\right.$ ) which is similar to the range of the four-momentum transfers squared $\left(150<Q^{2}<5000 \mathrm{GeV}^{2}\right)$ of the event sample. The cross sections are of the same size for both jet algorithms and show a slightly harder $E_{T}$ spectrum with increasing $Q^{2}$. The hadronization corrections are seen to be below $10 \%$ for both algorithms. The ratio of data and theoretical prediction is shown in Fig. 5. Over the whole range of $E_{T}$ and $Q^{2}$ the NLO calculation, corrected for hadronization effects, gives a good description of the data.

\subsection{Dijet Cross Section}

The inclusive dijet cross section is measured over the large range of four-momentum transfers squared $5<Q^{2}<15000 \mathrm{GeV}^{2}$ using the inclusive $k_{\perp}$ jet algorithm. At high $Q^{2}$ additional measurements have been made using the three other jet algorithms.

The dijet cross section for the inclusive $k_{\perp}$ algorithm is shown in Fig. 6 as a function of $Q^{2}$ in the range $5<Q^{2}<15000 \mathrm{GeV}^{2}$ for the central analysis cut $\left(E_{T 1}+E_{T 2}>17 \mathrm{GeV}\right)$ and an additional harder cut $\left(E_{T 1}+E_{T 2}>40 \mathrm{GeV}\right)$ on the sum of the transverse energies of 


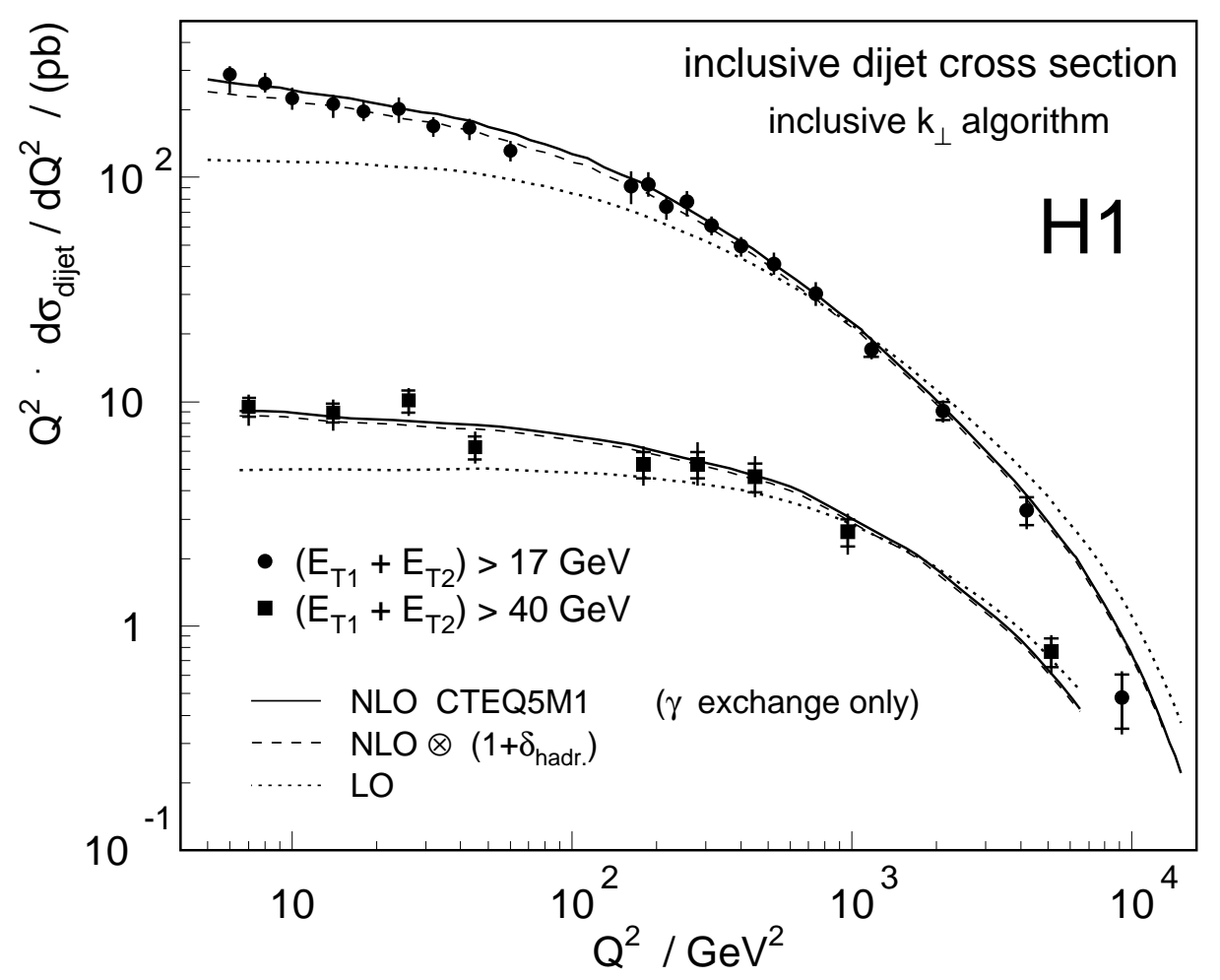

Figure 6: The dijet cross section measured with the inclusive $k_{\perp}$ algorithm as a function of $Q^{2}$ for different cuts on the sum of the transverse jet energies. The data are compared to the perturbative QCD prediction in NLO (solid line), in LO (dotted line) and to a theoretical prediction where hadronization corrections are added to the NLO prediction (dashed line).

the two jets with highest $E_{T}$. The data are compared to the NLO prediction without and with hadronization corrections applied, as well as to a $\mathrm{LO}$ calculation.

The hadronization corrections are small and increase only slightly towards lower $Q^{2}$. The NLO prediction, including hadronization effects, gives a good description of the data over the large phase space in $E_{T}$ and $Q^{2}$ and nicely models the reduced $Q^{2}$ dependence observed for the higher $E_{T}$ data. Deviations at $Q^{2} \simeq 10000 \mathrm{GeV}^{2}$ can be attributed to the neglect of $Z^{\circ}$ exchange in the calculation.

The dijet cross section at high $Q^{2}$, measured using all four jet algorithms mentioned above, is shown in Fig. Q 7. A different $Q^{2}$ dependence is observed for the inclusive and the exclusive algorithms which is a reflection of the different jet selection criteria and which is well reproduced by the theory. While hadronization corrections have only a small effect for the inclusive jet algorithms, they lower the NLO predictions for the exclusive algorithms by up to $30 \%$ at $Q^{2}=150 \mathrm{GeV}^{2}$. However, when these non-perturbative corrections are included the dijet cross sections are in all four cases well described by the theoretical curves.

In the following more details of the dijet distributions are given. For these studies we restrict the phase space to $Q^{2}<5000 \mathrm{GeV}^{2}$ in order to avoid the region where contributions from $Z^{\circ}$

\footnotetext{
${ }^{8}$ All cross sections have been measured as bin-averaged cross sections and all but two are presented this way, the only exception being the presentation of the $Q^{2}$ dependence in Figs. 6 and 7 . Here, to compare the data to the differential NLO prediction the points are presented at the bin-center, as determined using the NLO calculation.
} 


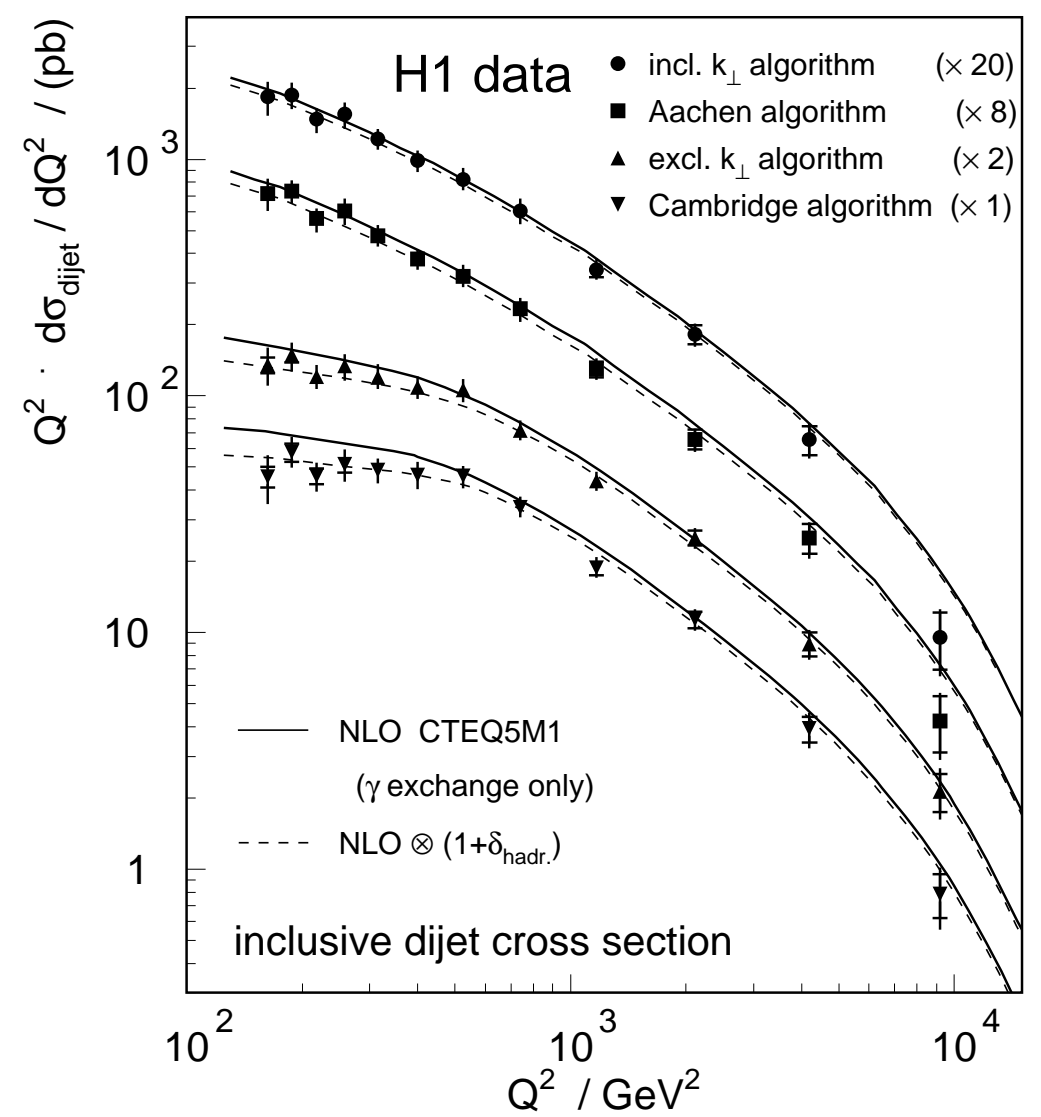

Figure 7: The dijet cross section as a function of $Q^{2}$ for four jet algorithms. The data are compared to the perturbative QCD prediction in NLO with (dashed line) and without (solid line) hadronization corrections included.

exchange are sizable. We present the double-differential dijet cross section as a function of the variables $Q^{2}, M_{\mathrm{jj}}, \bar{E}_{T}, \eta^{\prime}, x_{p}, \xi$ and $\eta_{\mathrm{forw}}$ lab in Figs. 8- 13. As for the $Q^{2}$ distribution in Fig. 7, the results are compared to the perturbative QCD prediction in NLO with and without hadronization corrections included. In Fig. 11 the contribution from gluon-induced processes is shown in addition.

The distributions of the invariant dijet mass $M_{\mathrm{jj}}$ and the average transverse jet energy $\bar{E}_{T}$ are shown in Fig. 8 covering a range of $15<M_{\mathrm{jj}}<95 \mathrm{GeV}$ and $8.5<\bar{E}_{T}<60 \mathrm{GeV}$. In both distributions we observe a harder spectrum towards larger $Q^{2}$. The NLO prediction, including hadronization corrections, gives a good overall description, except at lowest $Q^{2}$ where it describes the shape, but not the magnitude, of the cross section. The increasing hardness of the $\bar{E}_{T}$ distribution at higher $Q^{2}$ is also seen for the other jet algorithms in Fig. $Q$.

The distribution of the pseudorapidity $\eta^{\prime}$ (as defined in section 2.3) is shown in Fig. 10 for different regions of $\bar{E}_{T}$ for the low and the high $Q^{2}$ data. In both data sets the fraction of jets produced centrally in the dijet center-of-mass frame is observed to be larger at higher $\bar{E}_{T}$.

The partonic scaling variable $x_{p}$ is defined as the ratio of the Bjorken scaling variable $x_{\mathrm{Bj}}$ and the reconstructed parton momentum fraction $\xi$. In the distribution shown in Fig. 111 (left) a strong variation of the $x_{p}$ range is seen. Towards lower $Q^{2}$ values $\xi$ differs by up to three 

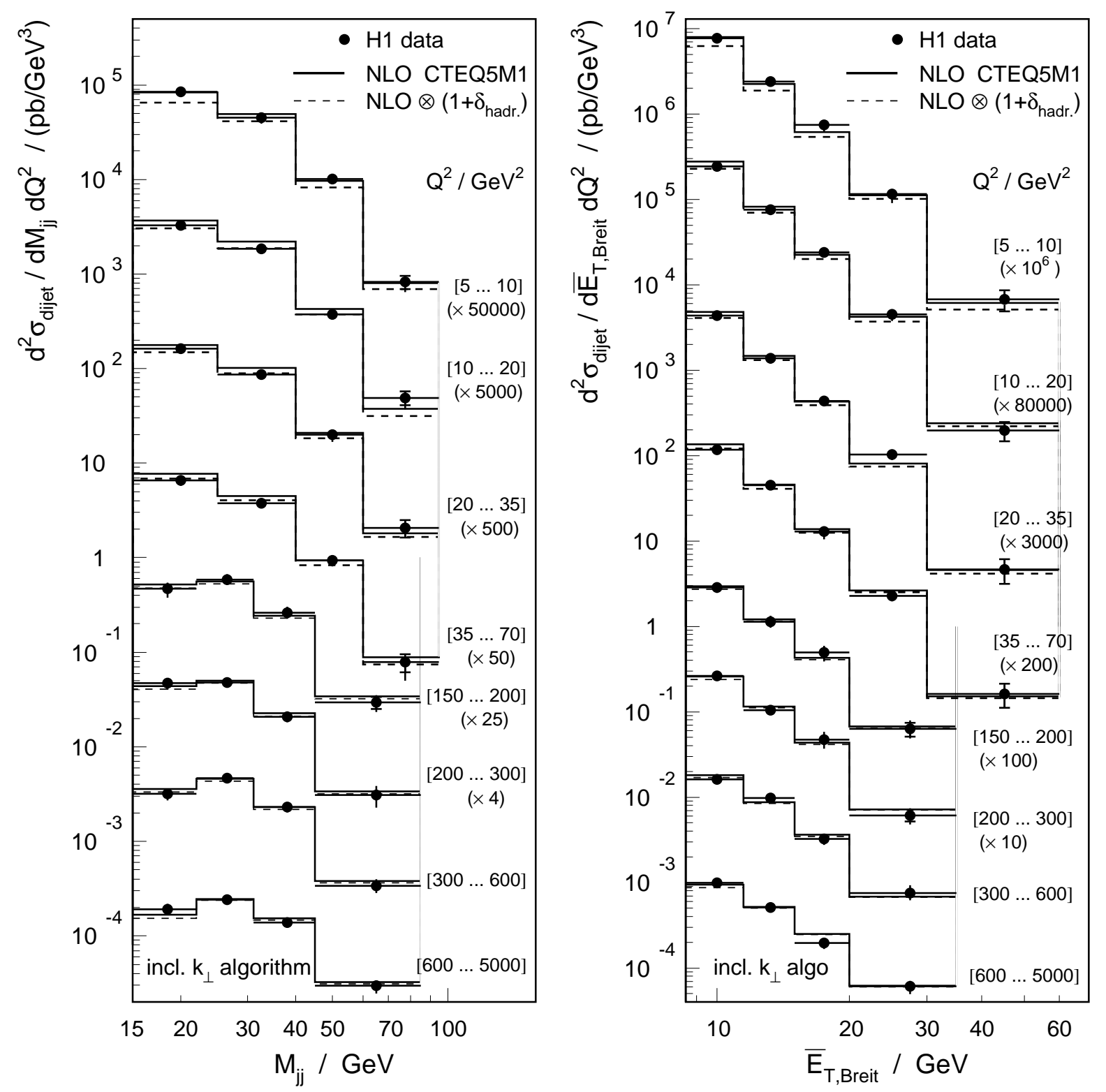

Figure 8: The dijet cross section for the inclusive $k_{\perp}$ algorithm as a function of $M_{\mathrm{jj}}$ (left) and $\bar{E}_{T}$ (right) in different regions of $Q^{2}$. The data are compared to the perturbative QCD prediction in NLO with (dashed line) and without (solid line) hadronization corrections included.

orders of magnitude from $x_{\mathrm{Bj}}$. At leading order the variable $\xi$ represents the fraction of the proton momentum carried by the struck parton. The dijet cross section in bins of $\xi$ is therefore directly proportional to the size of the parton densities at the parton momentum fraction $x=\xi$. Fig. 11 (right) shows the $\xi$ distribution in different regions of $Q^{2}$. The dijet data are seen to be sensitive to partons with momentum fractions $0.004 \lesssim \xi \lesssim 0.3$ which only increase slightly with increasing $Q^{2}$. The $\xi$ distribution is of special importance in the QCD analysis for the determination of the gluon density in the proton. Therefore we display the contribution from gluon induced processes to this distribution which varies strongly from $\simeq 80 \%$ at low $Q^{2}$ to $\simeq 40 \%$ at the highest $Q^{2}$. Both, the $\xi$ and the $x_{p}$ distributions are well described by the NLO calculation over the whole range of $Q^{2}$, independent of the fractional gluon contribution. 

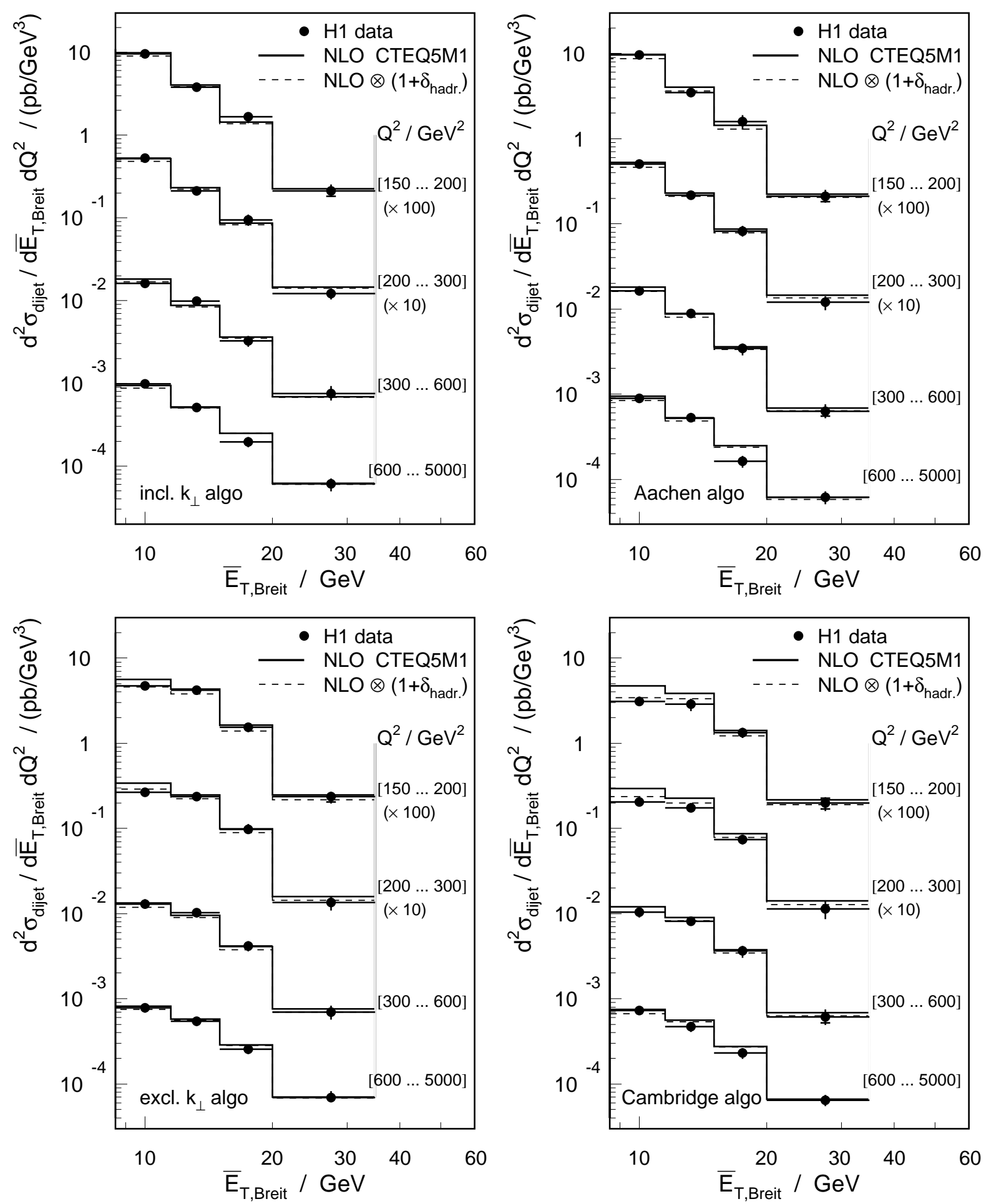

Figure 9: The dijet cross section as a function of the average transverse jet energy in the Breit frame in different regions of $Q^{2}$ for the inclusive $k_{\perp}$ algorithm (top left), the Aachen algorithm (top right), the exclusive $k_{\perp}$ algorithm (bottom left) and the Cambridge algorithm (bottom right). The data are compared to the perturbative QCD prediction in NLO with (dashed line) and without (solid line) hadronization corrections included. 

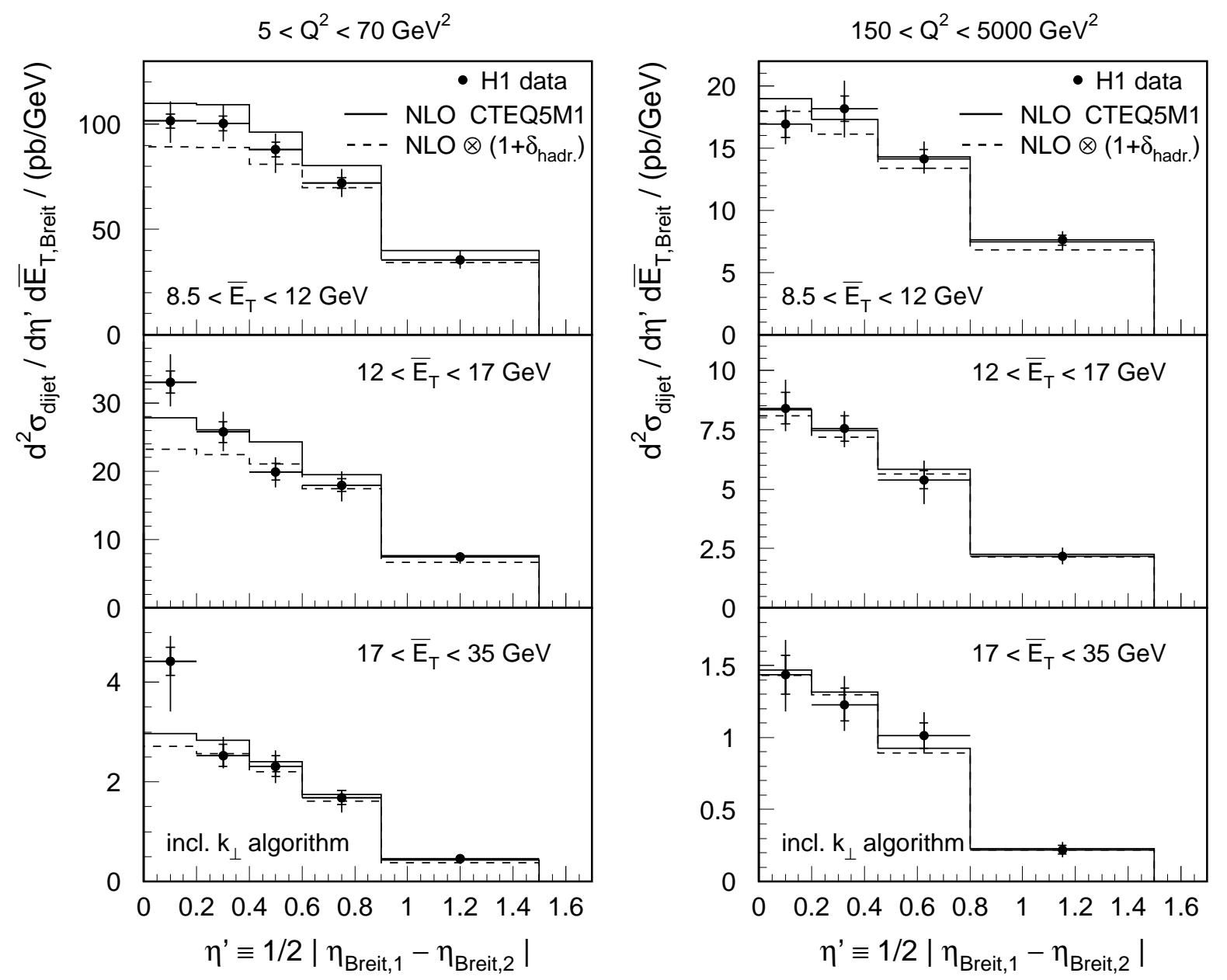

Figure 10: The dijet cross section for the inclusive $k_{\perp}$ algorithm as a function of the variable $\eta^{\prime}$ in different regions of $\bar{E}_{T}$ at low $Q^{2}$ (left) and at high $Q^{2}$ (right). The data are compared to the perturbative QCD prediction in NLO with (dashed line) and without (solid line) hadronization corrections included.

The $\xi$ distribution is also presented for the other jet algorithms (Fig. 12). While the distributions for the inclusive jet algorithms (incl. $k_{\perp}$ and Aachen) are already described by the NLO calculation (without hadronization corrections), large deviations are seen for the exclusive algorithms (excl. $k_{\perp}$ and Cambridge), especially at small $\xi$ corresponding to small dijet masses. However, in this region hadronization corrections are very large for the exclusive algorithms. Within the estimated size of these corrections theory and data are consistent, except in those regions where the corrections are especially large.

Fig. 13 finally shows the distribution of the forward jet $\eta_{\text {forw, lab }}$ in the laboratory frame in different regions of $Q^{2}$. While at larger $Q^{2}$ the distribution is seen to decrease towards the cut value at $\eta_{\text {forw, lab }}=2.5$, it is flatter at low $Q^{2}$. The theoretical calculation gives a reasonable description of this angular distribution. In addition also the LO prediction is included. Although the NLO corrections become large in the forward region (i.e. at large $\eta_{\text {forw, lab }}$ ) towards lower $Q^{2}$, the NLO calculation does describe the data remarkably well. Only at lowest $Q^{2}$ the NLO calculation clearly fails to describe the data, which is in agreement with the observations made in an earlier analysis of forward jet production [35]. 

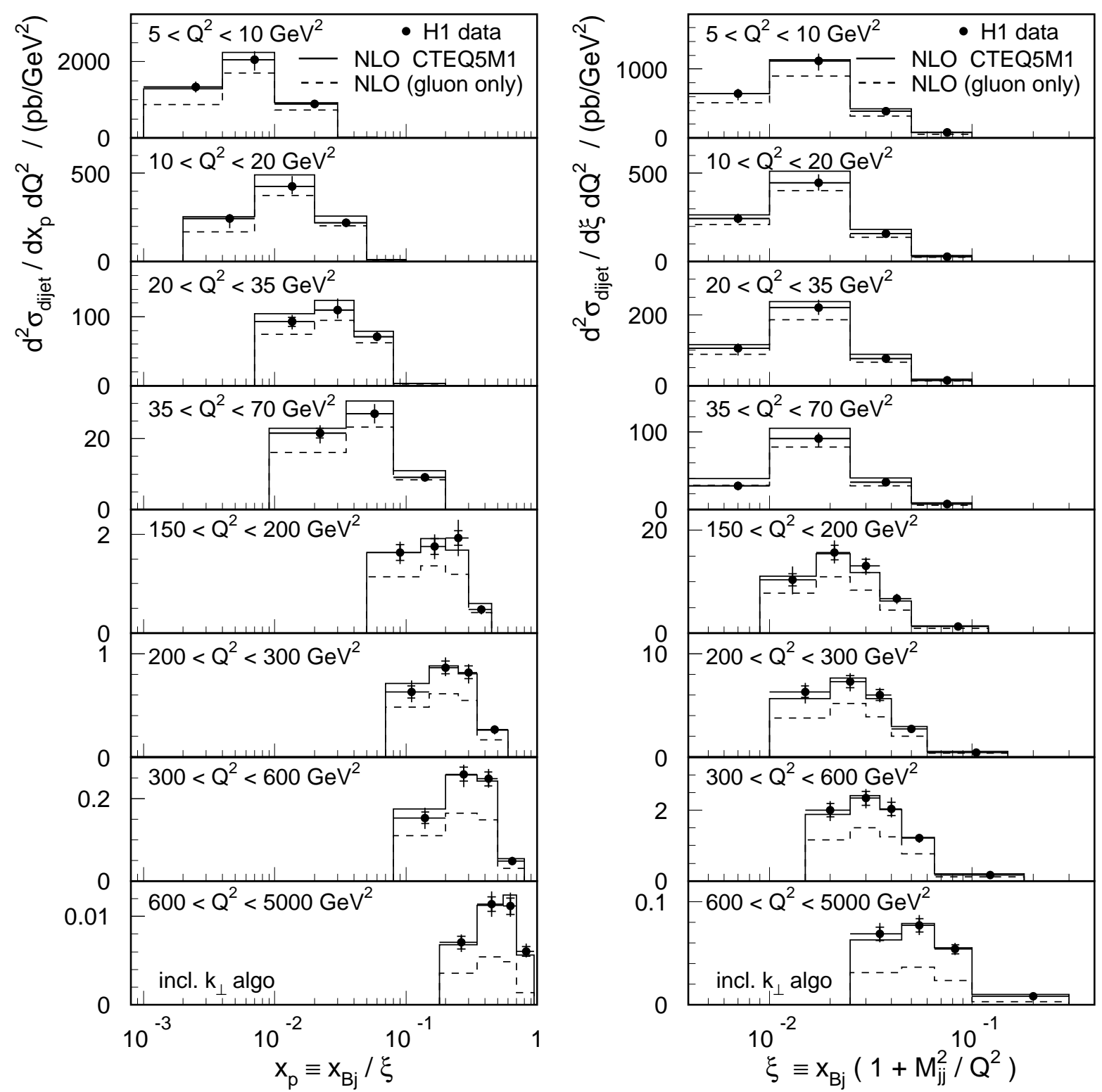

Figure 11: The dijet cross section for the inclusive $k_{\perp}$ algorithm as a function of the variables $x_{p}$ (left) and $\xi$ (right). The perturbative QCD prediction in NLO (solid line) is compared to the measured dijet cross section. In addition the contribution from gluon induced processes is shown (dashed line).

The perturbative NLO prediction gives a good description of the data for those observables for which NLO corrections and non-perturbative contributions are small. This agreement is seen in all regions of phase space, independent of whether they are dominated by the QCDCompton or the boson-gluon fusion processes. For observables with not too large hadronization corrections the differences between the perturbative calculation and the data can be explained by the predictions of phenomenological hadronization models. In the kinematic region of $10<$ $Q^{2}<70 \mathrm{GeV}^{2}$ theory still gives a good description of the data although NLO corrections become large. The theoretical calculations only fail at $Q^{2}<10 \mathrm{GeV}^{2}$ where NLO corrections are largest (with $k$-factors above two), such that contributions beyond NLO are expected to be sizable. 

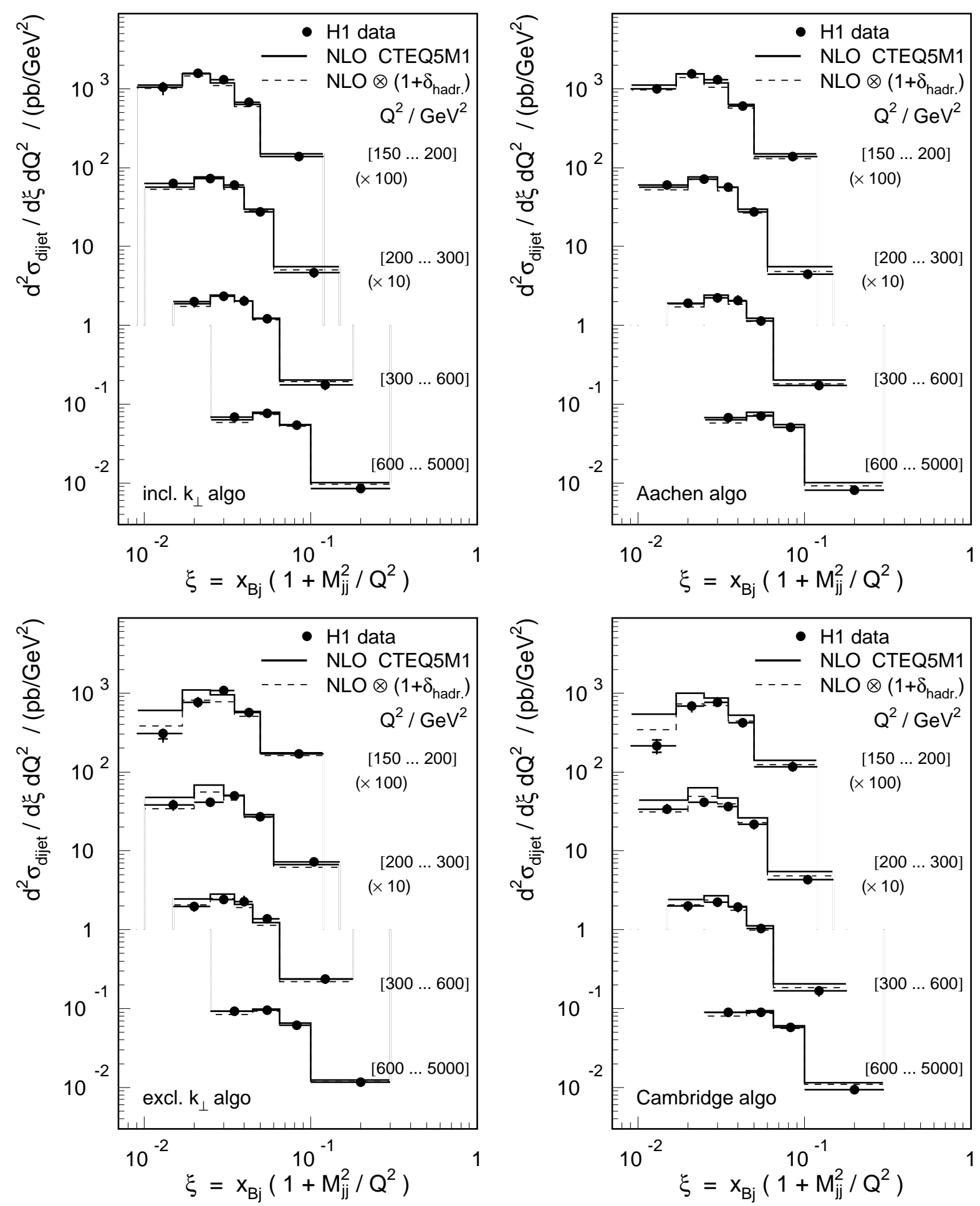

Figure 12: The dijet cross section as a function of the reconstructed parton momentum fraction $\xi$. The data are measured in different regions of $Q^{2}$ for the inclusive $k_{\perp}$ algorithm (top left), the Aachen algorithm (top right), the exclusive $k_{\perp}$ algorithm (bottom left) and the Cambridge algorithm (bottom right). The data are compared to the perturbative QCD prediction in NLO with (dashed line) and without (solid line) hadronization corrections included. 


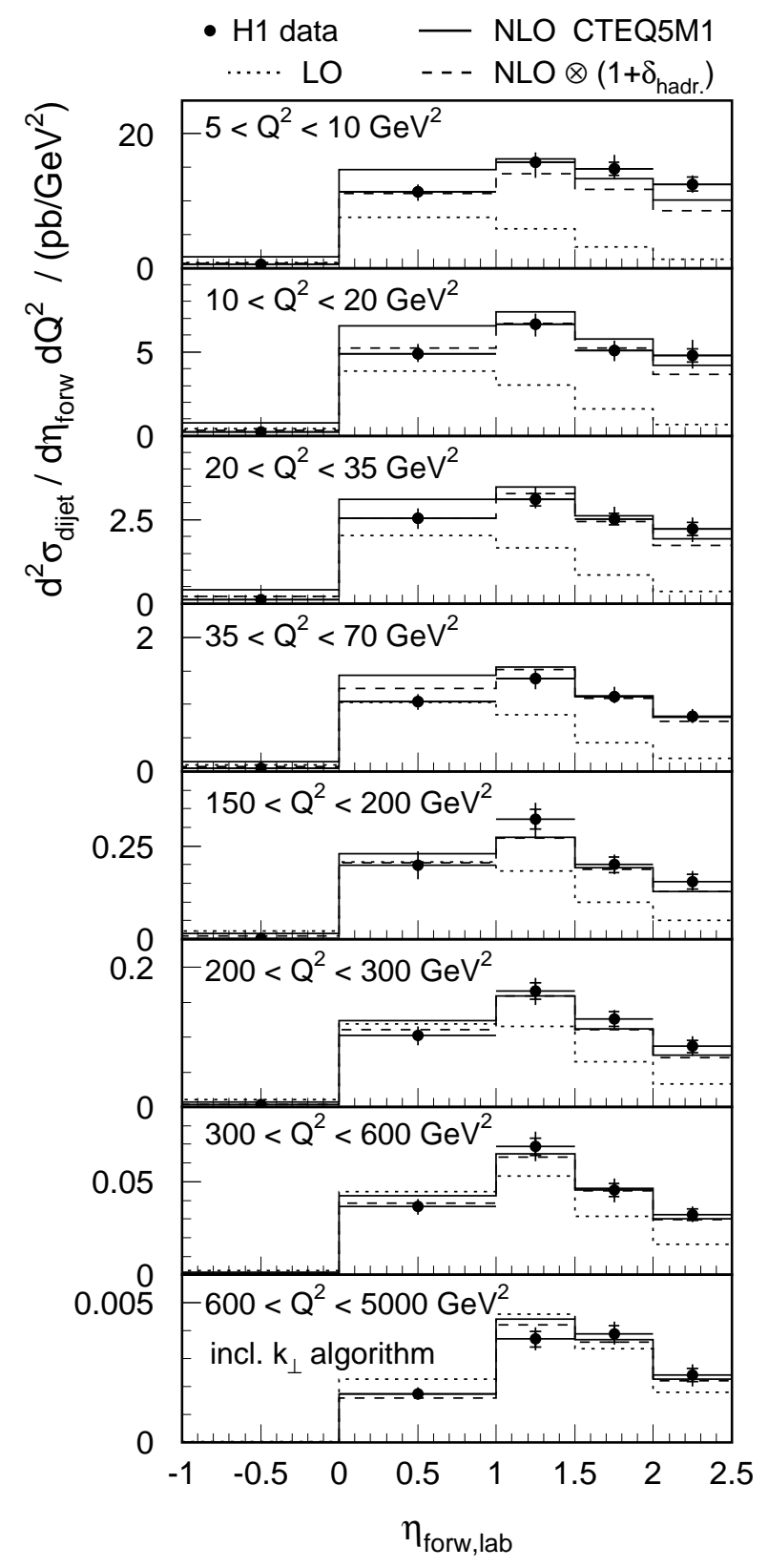

Figure 13: The dijet cross section for the inclusive $k_{\perp}$ algorithm as a function of the pseudorapidity of the forward jet in the laboratory frame. The data are compared to the perturbative QCD prediction in NLO (solid line), in LO (dotted line) and to a theoretical prediction where hadronization corrections are added to the NLO calculation (dashed line). 


\section{QCD Analysis}

The QCD predictions depend primarily on $\alpha_{s}$ and on the gluon and the quark density functions of the proton. In this section we present QCD analyses of the data in which we determine these parameters of the theory. We briefly discuss how different processes in DIS are directly sensitive to the different parameters and introduce the physical and technical assumptions with which the QCD fits are performed.

\subsection{Strategy}

In perturbative QCD (pQCD) the cross section of any process in deep-inelastic lepton-proton scattering can be written as a convolution of (process specific) perturbative coefficients $c_{a, n}$ with (universal) parton density functions $f_{a / p}$ of the proton

$$
\sigma=\sum_{a, n} \int_{0}^{1} \mathrm{~d} x \alpha_{s}^{n}\left(\mu_{r}\right) c_{a, n}\left(\frac{x_{\mathrm{Bj}}}{x}, \mu_{r}, \mu_{f}\right) f_{a / p}\left(x, \mu_{f}\right) .
$$

The sum runs over all contributing parton flavors $a$ (quarks and gluon) and all orders $n$ considered in the perturbative expansion. The integration is carried out over all fractional parton momenta $x$. The coefficients $c_{a, n}$ are predicted by pQCD. They are currently known to nextto-leading order in the strong coupling constant for the inclusive DIS cross section $(n=0,1)$ and for the dijet and the inclusive jet cross section $(n=1,2)$ [36]. In the regions of sufficiently large transverse jet energies and not too large values of $Q^{2}\left(Q^{2}<5000 \mathrm{GeV}^{2}\right)$ the effects of $Z^{\circ}$ exchange and of quark masses (for five quark flavors) can be neglected as shown in [5] using the program MEPJET [37]. In this approximation the perturbative coefficients of the quarks for the inclusive DIS cross section and for the jet cross sections fulfill the relations

$$
c_{u}=c_{c}=c_{\bar{u}}=c_{\bar{c}} \quad \text { and } \quad c_{d}=c_{s}=c_{b}=c_{\bar{d}}=c_{\bar{s}}=c_{\bar{b}}
$$

in each order of $\alpha_{s}$. Therefore only three coefficients are independent and the cross section in (8) can be described by three independent parton density functions $9 x G(x), x \Delta(x)$ and $x \Sigma(x)$ with coefficients $c_{G}, c_{\Delta}$ and $c_{\Sigma}$, which have to be defined such that

$$
c_{g} g(x)+\sum_{a} c_{a}\left(q_{a}(x)+\bar{q}_{a}(x)\right)=c_{G} G(x)+c_{\Sigma} \Sigma(x)+c_{\Delta} \Delta(x),
$$

where the sums run over all quark flavors $a$. These three parton density functions are chosen to be

$$
\begin{aligned}
\text { Gluon: } & x G(x) \equiv x g(x), \\
\text { Sigma: } & x \Sigma(x) \equiv x \sum_{a}\left(q_{a}(x)+\bar{q}_{a}(x)\right), \\
\text { Delta: } & x \Delta(x) \equiv x \sum_{a} e_{a}^{2}\left(q_{a}(x)+\bar{q}_{a}(x)\right),
\end{aligned}
$$

\footnotetext{
${ }^{9}$ We do not explicitly display the dependence on the factorization scale.
} 


\begin{tabular}{|l|c|c|}
\hline & LO & NLO \\
\hline$\sigma_{\text {incl. DIS }}$ & $x \Delta(x)$ & $x \Delta(x), x G(x)$ \\
$\sigma_{\text {jets }}$ & $x G(x), x \Delta(x)$ & $x G(x), x \Delta(x), x \Sigma(x)$ \\
\hline
\end{tabular}

Table 1: Overview of the parton density functions contributing to different cross sections in LO and NLO.

where $e_{a}$ denotes the electric charge of the corresponding quark. The three corresponding coefficients are given by linear combinations of the single flavor coefficients

$$
c_{G}=c_{\text {gluon }} \quad c_{\Sigma}=\frac{1}{3}\left(4 c_{d}-c_{u}\right) \quad c_{\Delta}=3\left(c_{u}-c_{d}\right) .
$$

At orders $\mathcal{O}\left(\alpha_{s}^{0}\right)$ and $\mathcal{O}\left(\alpha_{s}^{1}\right)$ the contributions from different quark flavors are proportional to their electric charge squared (i.e. $c_{u}=4 c_{d}$ ). Therefore the coefficient $c_{\Sigma}$ in (12) vanishes and the only quark contributions to the cross sections come from $x \Delta(x)$. The gluon gives contributions at order $\mathcal{O}\left(\alpha_{s}^{1}\right)$ and higher. $x \Sigma(x)$ starts to contribute at order $\mathcal{O}\left(\alpha_{s}^{2}\right)$ and does therefore not enter the inclusive DIS cross section to next-to-leading order. Table 1 gives an overview of the orders in which the parton densities contribute to the different processes (to NLO). $x \Sigma(x)$ enters only the jet cross sections via the NLO corrections. At large $Q^{2}$ the contributions from $x \Sigma(x)$ are, however, small (4.5\% for the dijet cross section at $150<Q^{2}<200 \mathrm{GeV}^{2}$, decreasing to $2 \%$ at $600<Q^{2}<5000 \mathrm{GeV}^{2}$ ). In the following the parameterization CTEQ5M1 is used to determine this contribution which is not regarded as a degree of freedom in the analysis. This is, however, only a weak assumption which will (due to the smallness of the contribution) not bias the result.

With this approximation the inclusive DIS cross section and the jet cross section now depend on three quantities which will be determined in this analysis: $\alpha_{s}$, the gluon density $x G(x)$ and the quark density $x \Delta(x)$. To demonstrate the basic sensitivity the leading order cross sections are written in the symbolic form

$$
\begin{array}{lrlll} 
& \text { inclusive DIS cross section: } \quad & \sigma_{\text {incl. DIS }} & \propto \Delta \\
\text { jet cross sections in DIS: } & \sigma_{\text {jet }} & \propto & \alpha_{s} \cdot\left(c_{G} G+c_{\Delta} \Delta\right) .
\end{array}
$$

These relations make clear that in DIS a direct determination of either $\alpha_{s}$ or the gluon density can never be performed without considering the correlation with the other quantity. Three strategies are used in the QCD fits which differ by the amount of external information included in the analysis.

1. Determination of $\alpha_{s}$ from jet cross sections: using the jet cross sections measured one can determine $\alpha_{s}$ assuming external knowledge on the parton distributions as provided by global data analyses.

2. Consistent determination of the gluon density $x G(x)$ and the quark density $x \Delta(x)$ : including data on the inclusive DIS cross section, which are directly sensitive to the quarks only, and assuming the world average value of $\alpha_{s}\left(M_{Z}\right)$ the information provided by the jet data can be used for a direct determination of the gluon density together with the quark densities via a simultaneous fit. 
3. Simultaneous determination of $\alpha_{s}$, the gluon density $x G(x)$ and the quark density $x \Delta(x)$ : if the jet cross sections are measured in different phase space regions $\left(\sigma_{\text {jet }}, \sigma_{\text {jet }}^{\prime}\right)$ with different sensitivity to the quark and the gluon contributions (i.e. where $c_{G}^{\prime} / c_{\Delta}^{\prime} \neq c_{G} / c_{\Delta}$ ) a simultaneous direct determination of all free parameters is possible when again additional inclusive DIS data are included.

\subsection{Fitting Technique}

A determination of theoretical parameters can only be performed in phase space regions where theoretical predictions are reliable. Although the perturbative NLO calculation gives a good description of the jet data down to $Q^{2}=10 \mathrm{GeV}^{2}$, the QCD analysis is restricted to the region where NLO corrections are small (with $k$-factors below 1.4), i.e. to the region of high $Q^{2}(150<$ $Q^{2}<5000 \mathrm{GeV}^{2}$ ). For the main analysis the jet cross sections measured for the inclusive $k_{\perp}$ algorithm are used for which hadronization corrections are smallest. Jet cross sections from other jet algorithms are used to test the stability of the results. The uncertainties of the jet data and their correlations are treated as described in section 3.4.

In the second and in the third step of the analysis data on the (reduced) inclusive DIS cross section are included to exploit their sensitivity to the quark densities in the proton. A subsample is taken of the recently published measurement [28] in the range $150 \leq Q^{2} \leq 1000 \mathrm{GeV}^{2}$. Since the present analysis uses the same experimental techniques the effects of the point to point correlated experimental uncertainties can be fully taken into account.

The fit of the theoretical parameters is performed in a $\chi^{2}$ minimization using the program MINUIT [38]. The definition of $\chi^{2}$ [39] fully takes into account all correlations of experimental and theoretical uncertainties. This $\chi^{2}$ definition has also been used in recent global data analyses [40, 41] and in a previous $\mathrm{H} 1$ publication [42]. The quoted uncertainties of the fit parameters are defined by the change of the parameter for which the $\chi^{2}$ of the fit is increased by one.

In the fitting procedure the perturbative QCD predictions in NLO for the inclusive DIS cross section are directly compared to the data, while the NLO predictions for the jet cross sections are corrected for hadronization effects before they are compared to the jet data:

$$
\begin{aligned}
\sigma_{\text {incl. DIS }}^{\mathrm{H} 1} & \longleftrightarrow \sigma_{\text {incl. DIS }}^{\mathrm{NLO}} \\
\sigma_{\text {jet }}^{\mathrm{H} 1} & \longleftrightarrow \sigma_{\text {jet }}^{\mathrm{NLO}} \cdot\left(1+\delta_{\text {hadr. }}\right) \quad \text { with } \quad \delta_{\text {hadr. }}=\frac{\sigma_{\text {jet }}^{\text {hadron }}-\sigma_{\text {jet }}^{\text {parton }}}{\sigma_{\text {jet }}^{\text {parton }}} .
\end{aligned}
$$

The hadronization corrections are determined as described in section 2.4 using the average value from the model predictions by HERWIG, LEPTO and ARIADNE. The uncertainty from the model and the parameter dependence of these predictions is always below 3\% [5, 8]. The uncertainty in the matching of the parton level (parton cascade and NLO calculation) is taken into account by increasing the quoted uncertainty in those regions where the hadronization corrections are large. In detail, the uncertainty of the hadronization correction for each bin of the jet cross sections is taken to be half the size of the correction, but at least $3 \%$. This uncertainty is assumed to be correlated between the theoretical predictions for all data points.

The renormalization scale $\mu_{r}$ in the NLO calculation is identified with the process specific hard scales in both processes. The inclusive DIS cross section is evaluated at $\mu_{r}=\sqrt{Q^{2}}$ and 
the jet cross sections are evaluated at $\mu_{r}=E_{T}$ (inclusive jet cross section) and $\mu_{r}=\bar{E}_{T}$ (dijet cross section). For the jet cross section an example is given of how the results change for an alternative choice, $\mu_{r}=\sqrt{Q^{2}}$. The strong coupling constant $\alpha_{s}\left(\mu_{r}\right)$ is parameterized in terms of its value at the scale $\mu_{r}=M_{Z}$ using the numerical solution of the renormalization group equation in 4-loop accuracy [4], 44].

In principle the arguments invoked in the choice of the renormalization scale $\mu_{r}$ also apply to the factorization scale $\mu_{f}$ for the inclusive DIS cross section and for the jet cross section. However, a different choice is made for the following reasons. The different parton flavors have been combined into three independent parton density functions $x G\left(x, \mu_{f}\right), x \Delta\left(x, \mu_{f}\right)$ and $x \Sigma\left(x, \mu_{f}\right)$ (section 5.1). These three parton densities are, however, only independent as long as no evolution between different scales $\mu_{f}$ is performed. The evolution of the gluon density is coupled to the evolution of $x \Sigma\left(x, \mu_{f}\right)$. Furthermore, since $x \Delta\left(x, \mu_{f}\right)$ is not an eigenstate of the DGLAP evolution operators, the evolution requires its decomposition into a non-singlet and a singlet (i.e. $x \Sigma\left(x, \mu_{f}\right)$ ). This introduces an additional dependence between the quark densities. To avoid mixing between the different parton densities the parton distributions are not evolved to different scales. Instead the perturbative calculations are carried out at a fixed value of the factorization scale $\mu_{f}=\mu_{0}$. The jet cross sections are sensitive to the parton distributions in the $x$-range $0.008 \lesssim x \lesssim 0.3$ (see Fig. 11). In this $x$-range the factorization scale dependence of the parton density functions is not large. In a next-to-leading order calculation the remaining $\mu_{f}$ dependence given by the DGLAP evolution equations is largely compensated by a corresponding term in the perturbative coefficients. The perturbative cross sections therefore depend only weakly on the choice of the factorization scale. The difference between using a fixed factorization scale $\mu_{0}$ and performing the full DGLAP evolution at a scale $\mu_{f}$ is of higher order in $\alpha_{s}$ than those considered. If the scale $\mu_{f}$ is close to the fixed scale these higher order terms which are proportional to $\ln \left(\mu_{f} / \mu_{0}\right)$ are small. Therefore a fixed value of the factorization scale of the order of the average transverse jet energies in the dijet and the inclusive jet cross section $\mu_{f}=\mu_{0}=\sqrt{200} \mathrm{GeV} \simeq\left\langle E_{T}\right\rangle$ is used. The subsample of the (reduced) inclusive DIS cross section $150 \leq Q^{2} \leq 1000 \mathrm{GeV}^{2}$ has been chosen such that the four-momentum transfer is also of the same order of magnitude $\sqrt{Q^{2}} \simeq \mu_{0}=\sqrt{200} \mathrm{GeV}$.

Both the renormalization and the factorization scale dependences of the cross sections each are considered as correlated theoretical uncertainties. Both scales are (separately) varied by a factor $x_{\mu}$ around their nominal values $\mu_{0}$ in the range $x_{\mu}=\left\{\frac{1}{2}, 2\right\}$ and the ratios $\frac{\sigma_{\mathrm{NLO}}\left(x_{\mu} \cdot \mu_{0}\right)}{\sigma_{\mathrm{NLO}}\left(\mu_{0}\right)}$ are taken as the corresponding uncertainties. Together with the uncertainty from the hadronization corrections they constitute the quoted theoretical uncertainty of the fit results.

During the $\chi^{2}$ minimization procedure in the fit the NLO calculations of the jet cross sections have to be performed iteratively for different values of $\alpha_{s}\left(M_{Z}\right)$ and for different parton density functions (the number of calculations used to obtain the present results and to study their stability is in the order of one million). Since standard computations of NLO jet cross sections are time consuming the method [5] is used of pre-convoluting the perturbative coefficients with suitably defined functions which can then be folded with the parton densities and $\alpha_{s}$ for a fast computation of the NLO cross section.

\footnotetext{
${ }^{10}$ It has been checked that in the range of scales considered in this analysis, $7 \mathrm{GeV}<\mu_{r}<M_{Z}$, the differences between the 2-, 3- and 4-loop solutions are always below 3 per mille.
} 


\subsection{Determination of $\alpha_{s}$}

As a first step the QCD predictions are fitted to the jet cross sections using parameterizations for the parton distributions from global fits. The single free parameter which is determined in the fits is the value of the strong coupling constant. All $\alpha_{s}$ fit results presented hereafter consider all experimental and theoretical uncertainties. The contribution of the uncertainties of the parton distributions to the uncertainty of $\alpha_{s}$ is discussed separately.

The value of $\alpha_{s}\left(M_{Z}\right)$ is obtained from a fit to the inclusive jet cross section measured double-differentially with the inclusive $k_{\perp}$ algorithm. For the result, we use the parton distributions from the CTEQ5M1 parameterization [34] and check the effects of other choices. The renormalization scale is chosen to be $\mu_{r}=E_{T}$ and the factorization scale is set to the fixed value of $\mu_{f}=\sqrt{200} \mathrm{GeV}$ (the average $E_{T}$ of the jet sample). The effect on $\alpha_{s}\left(M_{Z}\right)$ of using a different choice for $\mu_{r}$ is studied.

The studies of the stability of the results include fits to the inclusive jet cross section measured with the Aachen jet algorithm, fits to the double-differential dijet cross section $\mathrm{d}^{2} \sigma_{\text {dijet }} / \mathrm{d} \bar{E}_{T} \mathrm{~d} Q^{2}$ using four different jet algorithms, and fits to other double-differential dijet distributions.

\section{Fits to Single Data Points}

Before carrying out combined fits to groups of data points the consistency of the data is tested by performing QCD fits separately to all sixteen single data points of the double-differential inclusive jet cross section.

The fit results are displayed in Fig. 14 for the four regions of $Q^{2}$. In each fit a result for $\alpha_{s}\left(E_{T}\right)$ is extracted which is presented at the average $E_{T}$ of the corresponding data point. The individual results are subsequently evolved to $\alpha_{s}\left(M_{Z}\right)$. Combined fits to all four data points in the same $Q^{2}$ regions are performed, leading to a combined result of $\alpha_{s}\left(M_{Z}\right)$ for each $Q^{2}$ region. The lower curves in the plots represent the combined fit results and their uncertainties and the three upper curves indicate the evolution of the combined result and its uncertainty according to the renormalization group equation. The single $\alpha_{s}\left(E_{T}\right)$ values are consistent with the predicted scale dependence of $\alpha_{s}$ and all combined $\alpha_{s}\left(M_{Z}\right)$ results are compatible with each other. The results obtained in the different $Q^{2}$ regions are (for $\mu_{r}=E_{T}$ )

$$
\begin{aligned}
150<Q^{2}<200 \mathrm{GeV}^{2}: & \left.\alpha_{s}\left(M_{Z}\right)=0.1225_{-0.0054}^{+0.0052} \text { (exp. }\right)_{-0.0062}^{+0.0060} \text { (th.) }, \\
200<Q^{2}<300 \mathrm{GeV}^{2}: & \alpha_{s}\left(M_{Z}=0.1202_{-0.0044}^{+0.0044} \text { (exp. }\right)_{-0.0056}^{+0.0052} \text { (th.) }, \\
300<Q^{2}<600 \mathrm{GeV}^{2}: & \left.\alpha_{s}\left(M_{Z}\right)=0.1198_{-0.0038}^{+0.0037} \text { (exp. }\right)_{-0.0046}^{+0.0040} \text { (th.) }, \\
600<Q^{2}<5000 \mathrm{GeV}^{2}: & \left.\alpha_{s}\left(M_{Z}\right)=0.1188_{-0.0048}^{+0.0048} \text { (exp. }\right)_{-0.0042}^{+0.0035} \text { (th.) } .
\end{aligned}
$$

While the experimental uncertainties are of similar size for all $\alpha_{s}\left(M_{Z}\right)$ values, the theoretical uncertainties shrink slightly towards larger $Q^{2}$. This is a consequence of the reduced renormalization scale dependence of the jet cross section at higher $Q^{2}$. 


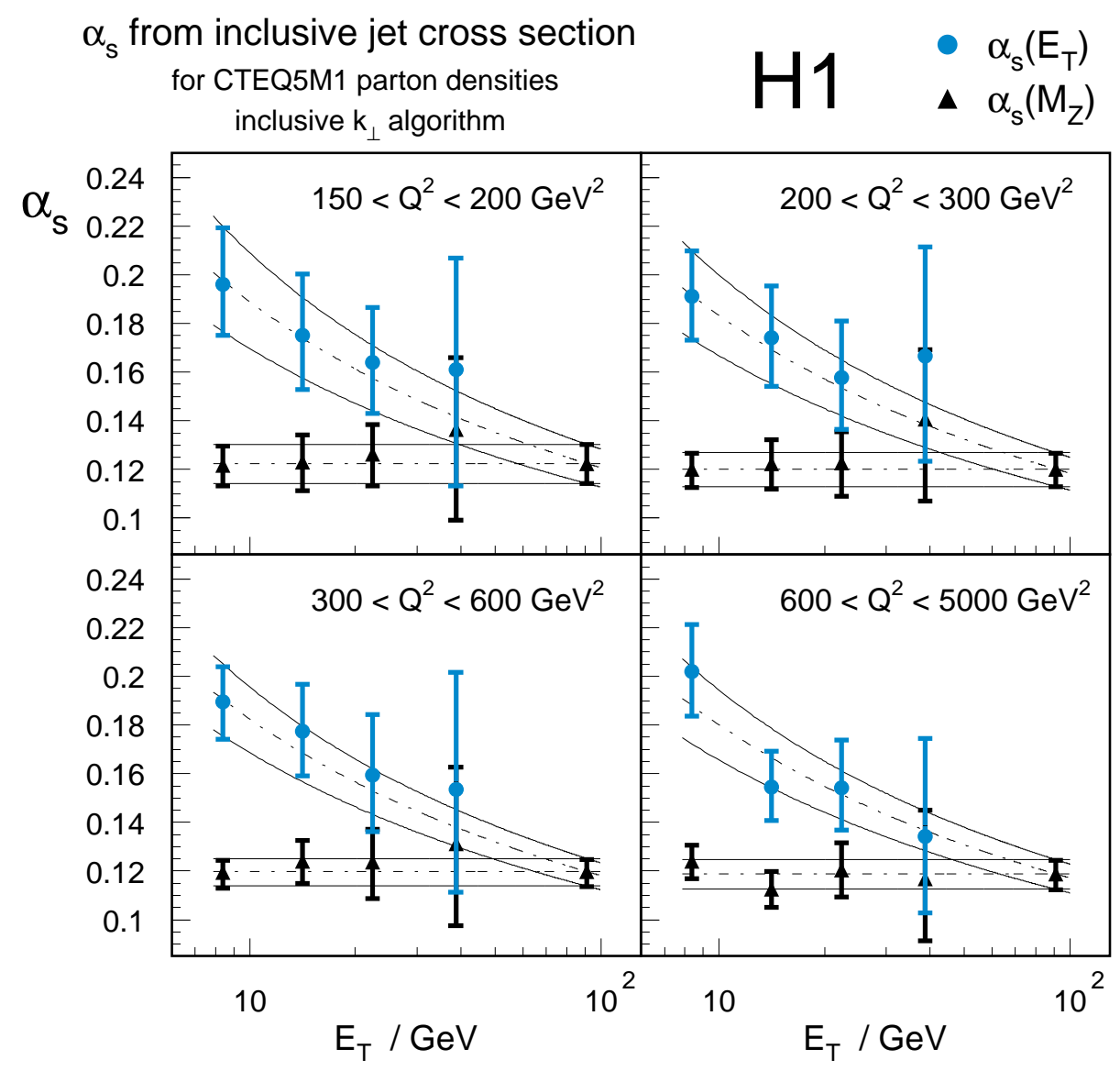

Figure 14: Determination of $\alpha_{s}$ from the inclusive jet cross section using the inclusive $k_{\perp}$ algorithm at a renormalization scale $\mu_{r}=E_{T}$. Displayed are the results of the fits to the single data points in each $Q^{2}$ region at each $E_{T}$ value (circles) including experimental and theoretical uncertainties. The single values are extrapolated to the $Z^{0}$ mass (triangles). A combined fit yields a result for $\alpha_{s}\left(M_{Z}\right)$ (rightmost triangle) for each $Q^{2}$ region. The lower curves represent the combined fit results and their uncertainties and the upper curves indicate the prediction of the renormalization group equation for their evolution.

\section{Combined Fit - Central $\alpha_{s}\left(M_{Z}\right)$ Result}

Having checked that the data are consistent over the whole range of $Q^{2}$ and $E_{T}$ combined fits are made to groups of data points. To study the $E_{T}$ dependence of $\alpha_{s}\left(E_{T}\right)$ the four data points of the same $E_{T}$ at different $Q^{2}$ are combined and four values of $\alpha_{s}\left(E_{T}\right)$ are extracted. The results are shown in Fig. 15. The four single values are evolved to $\alpha_{s}\left(M_{Z}\right)$. A combined fit to all 16 data points gives $\chi^{2} /$ n.d.f. $=3.80 / 15$ which is rather small, possibly reflecting a conservative estimate of systematic uncertainties. The central result is

$$
\left.\alpha_{s}\left(M_{Z}\right)=0.1186 \pm 0.0030 \text { (exp. }\right)_{-0.0045}^{+0.0039}(\text { th. }) \quad\left(\mu_{r}=E_{T}\right),
$$

in good agreement with the current world average of $\alpha_{s}\left(M_{Z}\right)=0.1184 \pm 0.0031$ [45]. The statistical uncertainty of the result is very small $( \pm 0.0007)$. The largest contribution to the experimental uncertainty comes from the hadronic energy scale of the LAr calorimeter. The 


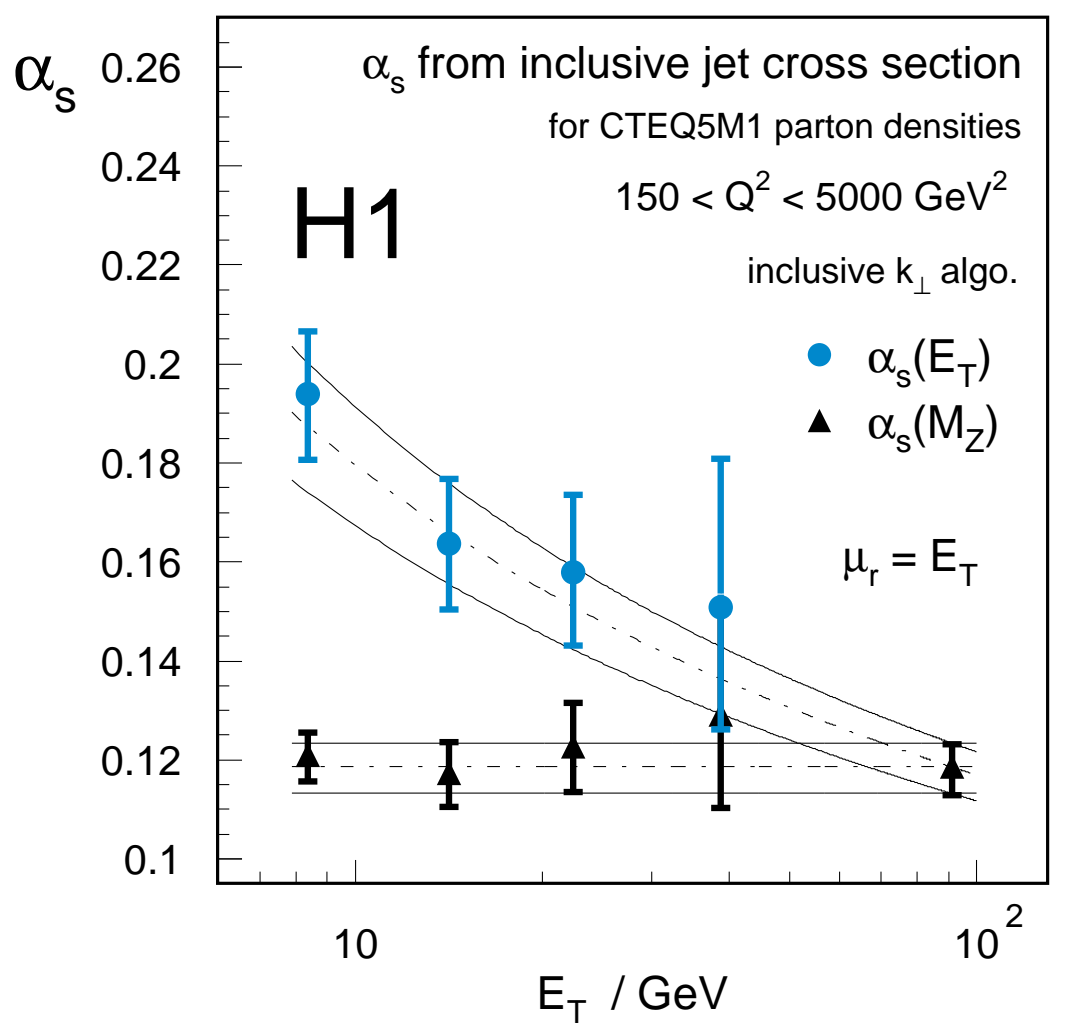

Figure 15: Determination of $\alpha_{s}$ from the inclusive jet cross section using the inclusive $k_{\perp}$ algorithm for the renormalization scale $\mu_{r}=E_{T}$. The results are shown for each $E_{T}$ value (circles) including experimental and theoretical uncertainties. The single values are extrapolated to the $Z^{0}$-mass (triangles). The final result for $\alpha_{s}\left(M_{Z}\right)$ (rightmost triangle) is obtained in a combined fit. The lower curves represent the combined fit result and its uncertainties and the upper curves indicate the prediction of the renormalization group equation for its energy evolution.

theoretical uncertainty includes equal contributions from the uncertainties of the hadronization corrections and the renormalization scale dependence. The contribution due to the uncertainty of the parton distributions is discussed below.

\section{Choice of $\sqrt{Q^{2}}$ as Renormalization Scale}

Another possible choice of the renormalization scale in the theoretical calculation is the fourmomentum transfer $\sqrt{Q^{2}}$. Analogous to the procedure applied before, an $\alpha_{s}$ determination is made for the renormalization scale $\mu_{r}=\sqrt{Q^{2}}$. A combined fit to the 16 data points gives $\chi^{2} /$ n.d.f. $=3.87 / 15$ and a result

$$
\left.\alpha_{s}\left(M_{Z}\right)=0.1227_{-0.0034}^{+0.0033} \text { (exp. }\right)_{-0.0060}^{+0.0055} \text { (th. } \quad\left(\mu_{r}=\sqrt{Q^{2}}\right) .
$$

Comparing this result with the one obtained for $\mu_{r}=E_{T}$ in (15), the central value is seen too be shifted by +0.0041 and the theoretical uncertainty to have increased substantially. This is due to the stronger renormalization scale dependence in the perturbative cross sections for $\mu_{r}=\sqrt{Q^{2}}$ compared to $\mu_{r}=E_{T}$. Within the increased uncertainty contribution from the renormalization scale dependence for $\mu_{r}=\sqrt{Q^{2}}$ both results are consistent. 


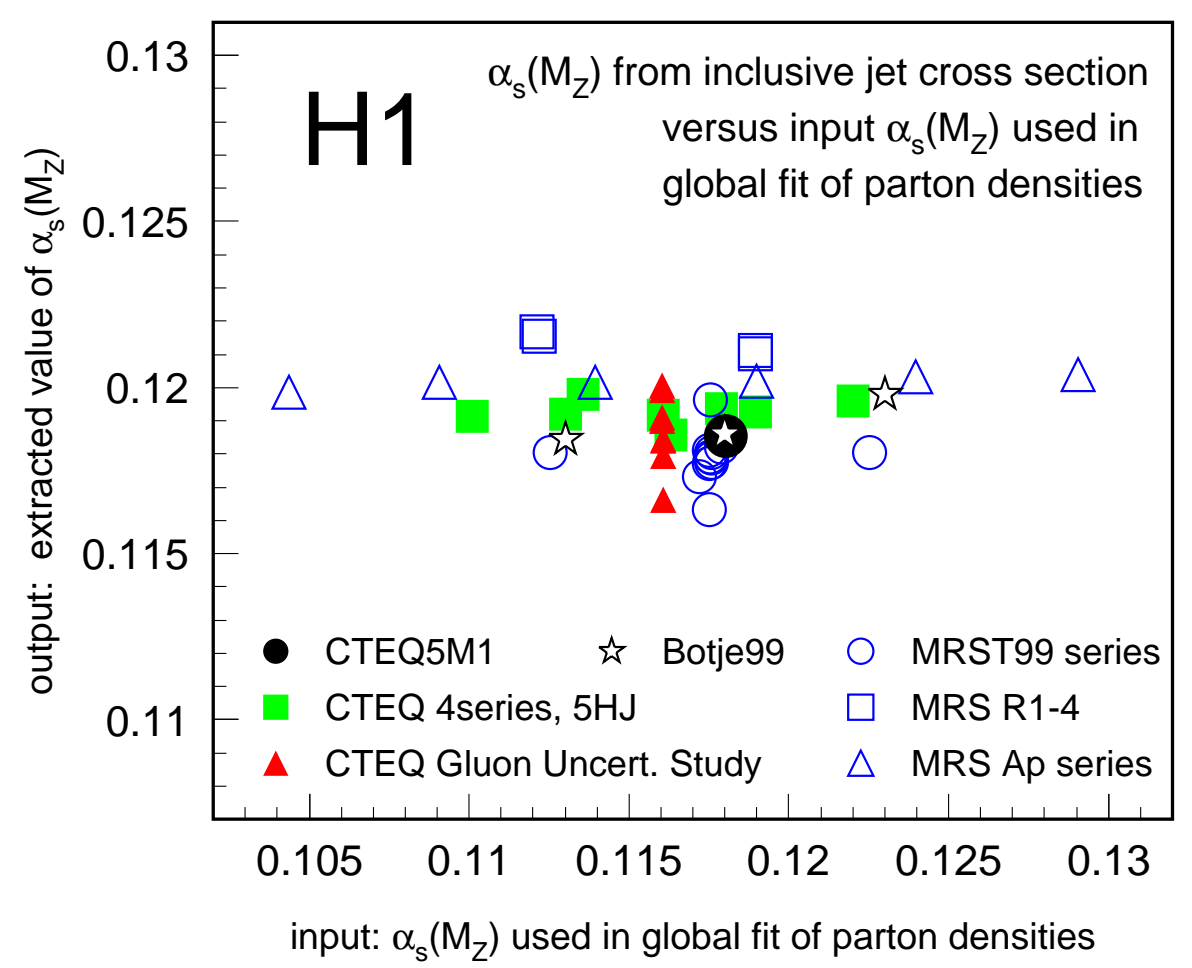

Figure 16: Dependence of the $\alpha_{s}\left(M_{Z}\right)$ fit result (for $\mu_{r}=E_{T}$ ) on the parton distributions used in the fit. The results are displayed as a function of the $\alpha_{s}\left(M_{Z}\right)$ value used in the corresponding global fit of the parton distributions. The correlation is shown for a comprehensive collection of different global fits.

\section{Using Different Parameterizations of Parton Distributions}

The central fit results are obtained for the parton distributions from the CTEQ5M1 parameterization [34]. The QCD fits are repeated using all parameterizations from recent global fits which have been performed in next-to-leading logarithmic accuracy in the $\overline{\mathrm{MS}}$ scheme. These include all sets from the fits CTEQ5 [34], Botje99 [40], MRST99 [46], CTEQ4 [47], MRSR [48], MRSAp [49] and the sets from the gluon uncertainty study [50] by the CTEQ collaboration $\square$. Many of these fits have provided sets of parton distributions for different assumptions for $\alpha_{s}\left(M_{Z}\right)$. Using these sets of parton distributions, the dependence of our results on the initially assumed $\alpha_{s}\left(M_{Z}\right)$ is studied.

The $\alpha_{s}\left(M_{Z}\right)$ results obtained for the different parton distributions are shown in Fig. 16 as a function of the $\alpha_{s}\left(M_{Z}\right)$ value used in the corresponding global fit. The range of the variations of the result is small and no significant correlation to the initially assumed $\alpha_{s}\left(M_{Z}\right)$ is seen. The largest deviations from the central result given in (15) are obtained with the MRSR3 parameterization $(+0.0031)$ and for the set MRST99 $(\mathrm{g} \downarrow)(-0.0022)$. Using the central parameterizations from the most recent analyses, results of $\alpha_{s}\left(M_{Z}\right)=0.1179$ for MRST99 and $\alpha_{s}\left(M_{Z}\right)=0.1186$ for Botje99 are obtained which are very close to the result obtained for CTEQ5M1.

\footnotetext{
${ }^{11}$ The parameterization from GRV98 [51] can not be used since the parameterizations of the charm and the bottom quark densities are not provided.
} 


\section{Uncertainties in the Parton Distributions}

A determination of the uncertainties of parton density functions (pdfs) has only recently become available [40]. This makes it possible to propagate these uncertainties into the predictions of physical quantities. While earlier attempts were restricted to a limited number of variations of single parton flavors [46, 50] the fit performed by Botje [40] does not only provide parameterizations of the central results, but also the covariance matrix $V_{i j}$ of the 28 fit parameters $p_{i}$ used, including the statistical and experimental systematic uncertainties. In addition further systematic studies were performed in [40] by repeating the fit under different physical assumptions; the corresponding deviations are, however, not included in the covariance matrix, but presented as single results. The combined information is used to determine the uncertainty of the $\alpha_{s}\left(M_{Z}\right)$ fit result by computing the contributions from the covariance matrix and the single systematic studies and add their contributions in quadrature. The uncertainty from the parton density functions is then given by

$$
\begin{aligned}
\Delta^{\mathrm{pdf}} \alpha_{s}\left(M_{Z}\right) & =\sqrt{\sum_{i, j} \frac{\partial \alpha_{s}\left(M_{Z}\right)}{\partial p_{i}} V_{i j} \frac{\partial \alpha_{s}\left(M_{Z}\right)}{\partial p_{j}}} \oplus \sqrt{\sum_{k}\left(\left(\Delta \alpha_{s}\left(M_{Z}\right)\right)_{k}^{\text {syst. }}\right)^{2}} \\
& = \pm 0.0019 \text { (pdf: stat. \& exp.) } \oplus{ }_{-0.0013}^{+0.0027} \text { (pdf: fit syst.) } \\
& ={ }_{-0.0023}^{+0.0033} \text { (pdf). }
\end{aligned}
$$

The largest single contribution comes from the factorization scale dependence which accounts for ${ }_{-0.0003}^{+0.0020}$ in $\Delta \alpha_{s}\left(M_{Z}\right)$. In fact, the uncertainty from the parton density functions, determined using this procedure is slightly larger than the spread observed in Fig. 16. The value from (17) is taken as the uncertainty of our $\alpha_{s}\left(M_{Z}\right)$ result due to the parton density functions. The final result is then

$$
\left.\alpha_{s}\left(M_{Z}\right)=0.1186 \pm 0.0030 \text { (exp. }\right)_{-0.0045}^{+0.0039}(\text { th. })_{-0.0023}^{+0.0033}(\mathrm{pdf}) \quad\left(\mu_{r}=E_{T}\right) .
$$

\section{Fits to Other Observables}

To test the stability of the central fit result the same QCD fits are made to some of the other jet distributions measured. Included are fits to the differential inclusive jet cross section $\mathrm{d}^{2} \sigma_{\text {jet }} / \mathrm{d} E_{T} \mathrm{~d} Q^{2}$ using the Aachen algorithm and the double-differential dijet cross section as a function of various variables for all four jet algorithms mentioned. For the latter the renormalization scale is chosen to be $\mu_{r}=\bar{E}_{T}$. The results of $\alpha_{s}\left(M_{Z}\right)$ from these fits including experimental, theoretical and the pdf uncertainties are displayed in Fig. 17. All $\alpha_{s}\left(M_{Z}\right)$ values are in good agreement with each other, with the central fit result given in (18) and with the current world average value. The results for the exclusive jet algorithms have larger theoretical uncertainties due to the larger hadronization corrections.

\subsection{Determination of the Gluon and the Quark Densities in the Proton}

The measurement of $\alpha_{s}$ described in section 5.3 depends on external knowledge of the parton content of the proton, and in particular on the uncertainty in the pdfs of the proton. The determined value of $\alpha_{s}$ is found to be consistent with measurements in which no initial state hadrons 


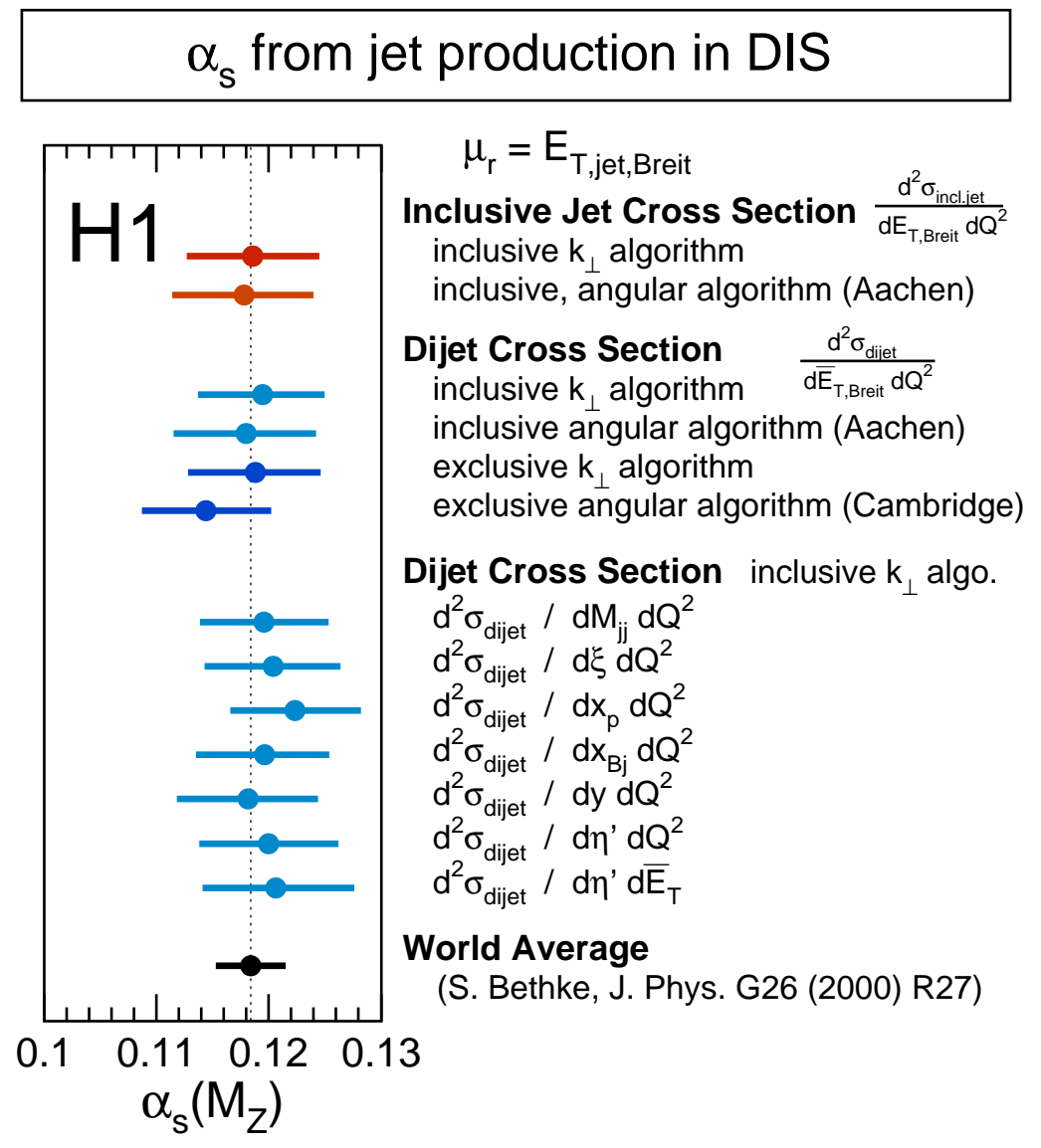

Figure 17: Comparison of $\alpha_{s}\left(M_{Z}\right)$ results from fits to different double-differential jet distributions.

are involved, for example in $e^{+} e^{-}$annihilation to hadrons [52]. The validity of pQCD at NLO in jet production in DIS is thereby demonstrated unequivocally to within the accuracy with which the strong coupling constant $\alpha_{s}$ is known.

It is therefore appropriate to pursue a determination of the parton density functions of the proton in NLO pQCD using measurements of jet production cross sections in DIS, assuming the value of the strong coupling constant $\alpha_{s}$ from external measurements. Such a determination is important for two reasons. First the measurement is in principle sensitive directly to both quark and gluon content in the proton, in contrast with studies of the evolution in $x_{\mathrm{Bj}}$ and $Q^{2}$ of the proton structure function $F_{2}$ where there is only direct sensitivity to the quark content. Second the range in the fractional momentum variable $\xi$ covered by a measurement using jets is different from that attained with $F_{2}$ measurements.

In the second step of the QCD analysis the sensitivity of the jet cross sections to the gluon density in the proton is exploited. The dijet cross section as a function of $\xi$ is directly sensitive to the gluon density at $x=\xi$. The inclusion of the inclusive jet cross section as a function of $E_{T}$ maximizes the accessible range in $x$. Data from a recent measurement of the inclusive DIS cross section [28] give strong, direct constraints on the quark density $x \Delta(x)$. Furthermore the strong coupling constant is set to the world average value $\alpha_{s}\left(M_{Z}\right)=0.1184 \pm 0.0031$ [45].

In the central fit the dijet cross section $\mathrm{d} \sigma_{\text {dijet }}^{2} / \mathrm{d} \xi \mathrm{d} Q^{2}$ at $150<Q^{2}<5000 \mathrm{GeV}^{2}$, the 


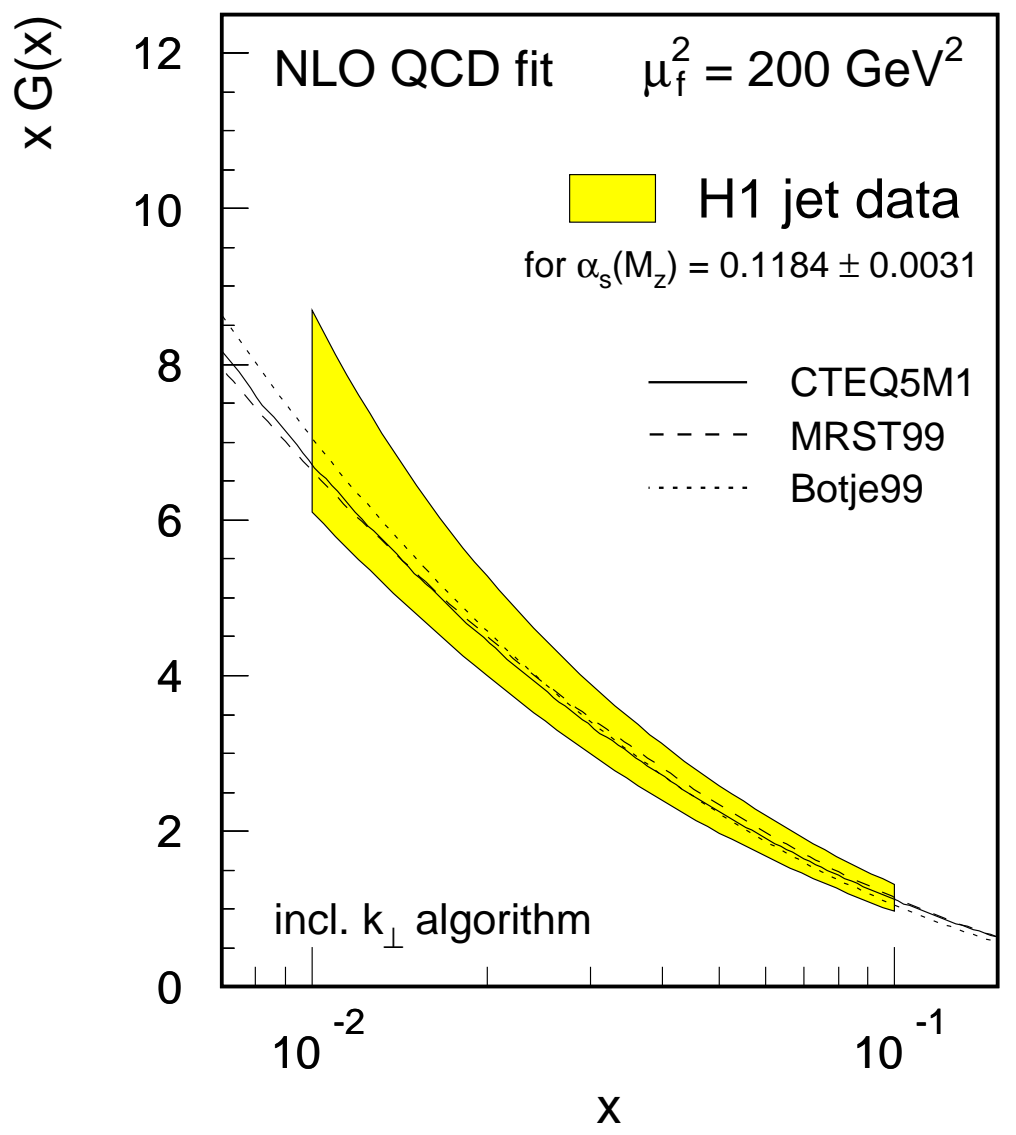

Figure 18: The gluon density $x G(x)$ in the proton, determined in a combined QCD fit to the inclusive DIS cross section, the inclusive jet cross section and the dijet cross section. The jet cross sections are measured using the inclusive $k_{\perp}$ jet algorithm. The error band includes the experimental and the theoretical uncertainties as well as the uncertainty of $\alpha_{s}\left(M_{Z}\right)$.

inclusive jet cross section $\mathrm{d} \sigma_{\text {jet }}^{2} / \mathrm{d} E_{T} \mathrm{~d} Q^{2}$ at $150<Q^{2}<5000 \mathrm{GeV}^{2}$ and the reduced inclusive DIS cross section $\tilde{\sigma}\left(x_{\mathrm{Bj}}, Q^{2}\right)$ from [28] in the range $150 \leq Q^{2} \leq 1000 \mathrm{GeV}^{2}\left(0.032<x_{\mathrm{Bj}}<\right.$ $0.65)$ are used. The gluon density and the $x \Delta(x)$ quark density are parameterized by

$$
x P(x)=A x^{b}(1-x)^{c}(1+d x)
$$

where $x P(x)$ stands for $x G(x)$ or $x \Delta(x)$.

The gluon density is determined in the range $0.01<x<0.1$ at the factorization scale $\mu_{f}=\sqrt{200} \mathrm{GeV}$ with $\chi^{2} /$ n.d.f. $=61.16 / 105$. The result is shown in Fig. 18. Displayed is the error band, including all experimental and theoretical uncertainties and the uncertainty from the value of the world average value of $\alpha_{s}\left(M_{Z}\right)$. The result is seen to be in good agreement with results from recent global data analyses. The integral of the gluon density over the range $0.01<x<0.1$ has been determined to be

$$
\begin{aligned}
\int_{0.01}^{0.1} \mathrm{~d} x x G\left(x, \mu_{f}^{2}=200 \mathrm{GeV}^{2}\right) & =0.229_{-0.030}^{+0.031} \text { (tot.), } \\
& \left.\left.=0.229_{-0.015}^{+0.016} \text { (exp. }\right)_{-0.021}^{+0.019} \text { (th. }\right)_{-0.015}^{+0.018}\left(\Delta \alpha_{s}\right) .
\end{aligned}
$$


This means that at the scale $\mu_{f}=\sqrt{200} \mathrm{GeV}$ gluons with a momentum fraction in the range $0.01<x<0.1$ carry $23 \%$ of the total proton momentum. This result is in good agreement with the results from global fits for which the integral has the values

CTEQ5M1: 0.227， MRST99: 0.232， GRV98HO: 0.235， Botje99: 0.227 .

The quark density $x \Delta\left(x, \mu_{f}^{2}=200 \mathrm{GeV}^{2}\right)$ determined in this fit is also close to results from global fits. To test the stability of the results various cross checks have been performed [5]:

(a) Different parameterizations of the parton densities:

$$
\begin{aligned}
3 \text { parameters } & x P(x)=A x^{b}(1-x)^{c} \\
5 \text { parameters (I) } & x P(x)=A x^{b}(1-x)^{c}\left(1+d x^{e}\right) \\
5 \text { parameters (II) } & x P(x)=A x^{b}(1-x)^{c}(1+d \sqrt{x}+e x)
\end{aligned}
$$

While the central result has been obtained using the 4-parameter ansatz in (19), the gluon density is unchanged when using other parameterizations and the quark density is stable if at least four parameters are used.

(b) Fits to subsets of the data: The fit has been applied to two subsamples of the data with $Q^{2}<300 \mathrm{GeV}^{2}$ and $Q^{2}>300 \mathrm{GeV}^{2}$ and both, the gluon and the quark results are unchanged.

(c) Fits to other jet distributions: The fits have been repeated using other jet distributions measured with the inclusive $k_{\perp}$ algorithm and also to jet distributions measured with other jet algorithms. In all cases the results are consistent with each other.

\subsection{Simultaneous Determination of $\alpha_{s}$ and the Proton pdfs}

In the above, $\alpha_{s}$ or the gluon density are extracted using external knowledge for the other. A more independent test of pQCD can be made in a simultaneous determination of both quantities. Such a determination has been performed by fitting the parton densities and $\alpha_{s}\left(M_{Z}\right)$ using the same data sets as in the previous section, the inclusive DIS cross section, the inclusive jet cross section $\mathrm{d}^{2} \sigma_{\text {jet }} / \mathrm{d} E_{T} \mathrm{~d} Q^{2}$ and the dijet cross section $\mathrm{d}^{2} \sigma_{\text {dijet }} / \mathrm{d} \xi \mathrm{d} Q^{2}$ (again measured with the inclusive $k_{\perp}$ algorithm). The gluon and the quark distributions are parameterized according to the 4-parameter formula in (19). The simultaneous fit yields $\chi^{2} /$ n.d.f. $=61.19 / 104$ and a result for the quark distributions identical to that which is obtained in the fit with a constrained $\alpha_{s}\left(M_{Z}\right)$. The results of this simultaneous fit are displayed in Fig. 19 as a correlation plot between $\alpha_{s}\left(M_{Z}\right)$ and the gluon density evaluated at four different values of $x=0.01,0.02,0.04,0.1$ which lie in the range where the jet cross sections are sensitive. The central fit result is indicated by the full marker and the error ellipse is the contour along which the $\chi^{2}$ of the fit is by one larger than the minimum (including experimental and theoretical uncertainties). The ellipticity of the contours indicate that the data included in this analysis are very sensitive to the product $\alpha_{s} \cdot x g(x)$ but do not allow a determination of both parameters simultaneously with useful precision. 


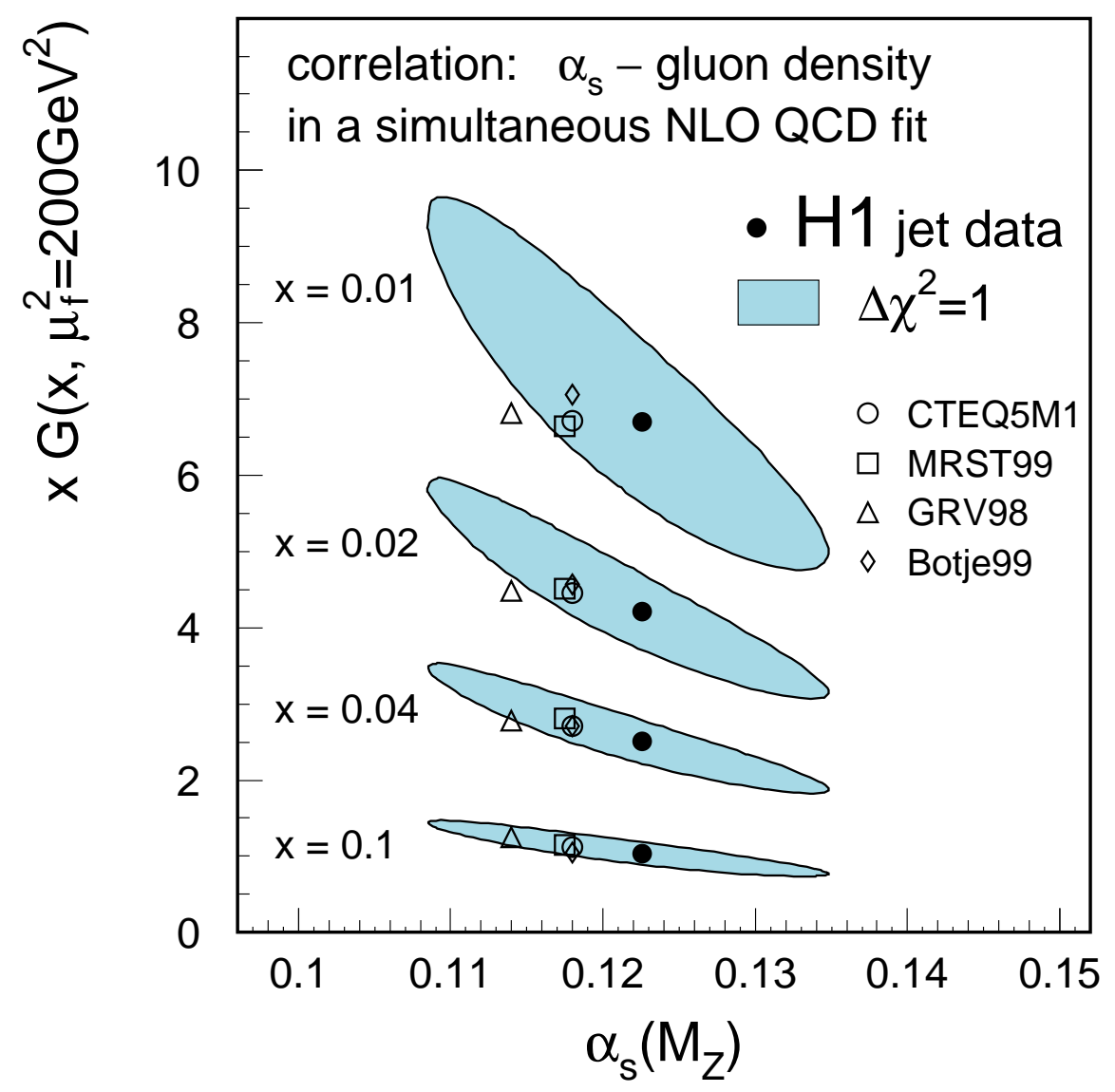

Figure 19: The correlation of the fit results for $\alpha_{s}\left(M_{Z}\right)$ and the gluon density at four different values of $x$, determined in a simultaneous QCD fit to the inclusive DIS cross section, the inclusive jet cross section and the dijet cross section. The jet cross sections are measured using the inclusive $k_{\perp}$ jet algorithm. The central fit result is indicated by the full marker. The error ellipses include the experimental and the theoretical uncertainties.

Also included in Fig. 19 are the results from global fits. All of these results are within the error ellipses except for GRV98 [51] (at $x<0.04$ ) which uses a relatively small value of $\alpha_{s}\left(M_{Z}\right)=0.114$.

The stability of the results in Fig. 19 has been tested in a similar way as already described in section 5.4. Fits have been performed excluding either the low $\left(<200 \mathrm{GeV}^{2}\right)$ or the high $\left(>600 \mathrm{GeV}^{2}\right.$ ) $Q^{2}$ data. Although the fits give consistent central results, the high $Q^{2}$ data are needed to achieve a stable determination of the contour of the error ellipsoid.

\section{Summary}

Jet production has been studied in the Breit frame in deep-inelastic positron-proton collisions at a center-of-mass energy of $\sqrt{s}=300 \mathrm{GeV}$. In the range of four-momentum transfers $5<Q^{2}<15000 \mathrm{GeV}^{2}$ and transverse jet energies $7<E_{T}<60 \mathrm{GeV}$ dijet and inclusive 
jet cross sections have been measured as a function of various variables using $k_{\perp}$ and angular ordered jet clustering algorithms. Perturbative QCD in next-to-leading order in $\alpha_{s}$ gives a good description of all observables for which next-to-leading order corrections are not too large and for which hadronization corrections are small. For those observables with moderately large hadronization corrections the deviations between data and the perturbative calculations are always consistent with the size of the hadronization corrections as predicted by phenomenological models. Only at $Q^{2}<10 \mathrm{GeV}^{2}$ do the theoretical predictions fail to describe the size of the measured jet cross sections. In this region, however, the NLO corrections are large, with $k$-factors above two indicating that NLO calculations are not reliable and that it is likely that the perturbative predictions receive large contributions from higher orders in $\alpha_{s}$ which can account for the observed difference.

QCD analyses of the data have been performed in the region of $Q^{2}>150 \mathrm{GeV}^{2}$, where NLO calculations are reliable, using the inclusive $k_{\perp}$ jet algorithm for which hadronization corrections are smallest. In a first step $\alpha_{s}$ has been determined in a fit to the inclusive jet cross section as a function of the transverse jet energy. Here the knowledge of the parton density functions of the proton is taken from the results of global fits. The observed $E_{T}$ dependence of $\alpha_{s}$ is consistent with the prediction of the renormalization group equation and a combined fit to the data yields

$$
\begin{aligned}
\alpha_{s}\left(M_{Z}\right) & =0.1186 \pm 0.0059 \text { (tot.) } \\
& =0.1186 \pm 0.0030 \text { (exp. })_{-0.0045}^{+0.0039}(\text { th. })_{-0.0023}^{+0.0033}(\text { pdf })
\end{aligned}
$$

This result is seen to be stable when the fit is performed to a variety of jet distributions measured with different jet algorithms.

Including $\mathrm{H} 1$ data on the inclusive neutral current DIS cross section, the jet data have been used for a consistent determination of the gluon density in the proton together with the quark densities. Setting $\alpha_{s}$ to the world average value [45] within its uncertainty of $\alpha_{s}\left(M_{Z}\right)=$ $0.1184 \pm 0.0031$ the gluon density is determined in the range of momentum fractions $0.01<$ $x<0.1$ at a factorization scale of the order of the transverse jet energies $\mu_{f}=\sqrt{200} \mathrm{GeV}$ in the $\overline{\mathrm{MS}}$ scheme. The integral over the range $0.01<x<0.1$ is determined to be

$$
\begin{aligned}
\int_{0.01}^{0.1} \mathrm{~d} x x G\left(x, \mu_{f}^{2}=200 \mathrm{GeV}^{2}\right) & =0.229_{-0.030}^{+0.031} \text { (tot.) } \\
& \left.=0.229_{-0.015}^{+0.016} \text { (exp.) }{ }_{-0.021}^{+0.019} \text { (th. }\right)_{-0.015}^{+0.018}\left(\Delta \alpha_{s}\right) .
\end{aligned}
$$

This result, as well as the differential distribution in $x$, are in good agreement with the results obtained in global fits.

Finally $\alpha_{s}$ and the gluon density in the proton have been determined simultaneously using data with direct sensitivity to both. The results and their uncertainties show a large anticorrelation. Here the single results of $\alpha_{s}$ and the gluon density have relatively large uncertainties, but the strong anticorrelation of the combined result clearly demonstrates the high sensitivity of the jet data to both. 


\section{Acknowledgments}

We wish to thank Erwin Mirkes, Dieter Zeppenfeld, Mike H. Seymour and Björn Pötter for many helpful discussions. We are grateful to the HERA machine group whose outstanding efforts have made and continue to make this experiment possible. We thank the engineers and technicians for their work in constructing and now maintaining the $\mathrm{H} 1$ detector, our funding agencies for financial support, the DESY technical staff for continual assistance, and the DESY directorate for the hospitality which they extend to the non-DESY members of the collaboration.

\section{References}

[1] H1 Collaboration, S. Aid et al., Nucl. Phys. B 449 (1995) 3;

ZEUS Collaboration, M. Derrick et al., Phys. Lett. B 363 (1995) 201;

H1 Collaboration, C. Adloff et al., Eur. Phys. J. C5 (1998) 625;

H1 Collaboration, C. Adloff et al., Eur. Phys. J. C6 (1999) 575.

[2] B.R. Webber, J. Phys. G19 (1993) 1567.

[3] S. Catani, Yu.L. Dokshitzer and B.R. Webber, Phys. Lett. B 285 (1992) 291.

[4] Yu.L. Dokshitzer, G.D. Leder, S. Moretti and B.R. Webber, JHEP 08 (1997) 001.

[5] M. Wobisch, PhD Thesis, RWTH Aachen (2000) PITHA 00/12.

[6] S.D. Ellis and D.E. Soper, Phys. Rev. D 48 (1993) 3160.

[7] S. Catani, Yu.L. Dokshitzer, M.H. Seymour and B.R. Webber, Nucl. Phys. B 406 (1993) 187.

[8] M. Wobisch and T. Wengler, Proceedings of the HERA Monte Carlo Workshop, eds. G. Grindhammer, G. Ingelman, H. Jung, T. Doyle, DESY-PROC-02-1999 (1999) 270.

[9] J. Huth et al., Proceedings of the Summer Study on High Energy Physics, Snowmass, Colorado (1990) 134.

[10] S. Frixione and G. Ridolfi, Nucl. Phys. B 507 (1997) 315.

[11] S. Catani and M.H. Seymour, Nucl. Phys. B 485 (1997) 291, Erratum-ibid. B 510 (1997) 503.

[12] C. Duprel, Th. Hadig, N. Kauer and M. Wobisch, Proceedings of the HERA Monte Carlo Workshop, eds. G. Grindhammer, G. Ingelman, H. Jung, T. Doyle, DESY-PROC-02-1999 (1999) 142.

[13] D. Graudenz, DISASTER++: Version 1.0 (October 1997), hep-ph/9710244.

[14] G. Marchesini et al., Comp. Phys. Comm. 67 (1992) 465.

[15] G. Ingelman, A. Edin and J. Rathsman, Comp. Phys. Comm. 101 (1997) 108. 
[16] H. Jung, Comp. Phys. Comm. 86 (1995) 147.

[17] L. Lönnblad, Comp. Phys. Comm. 71 (1992) 15.

[18] B.R. Webber, Nucl. Phys. B 238 (1984) 492.

[19] B. Andersson et al., Phys. Rep. 97 (1983) 31.

[20] A. Kwiatkowski, H. Spiesberger and H.-J. Möhring, Comp. Phys. Comm. 69 (1992) 155.

[21] K. Charchula, G. Schuler and H. Spiesberger, Comp. Phys. Comm. 81 (1994) 381.

[22] T. Carli, Proceedings of the HERA Monte Carlo Workshop, eds. G. Grindhammer, G. Ingelman, H. Jung, T. Doyle, DESY-PROC-02-1999 (1999) 185.

[23] H1 Collaboration, I. Abt et al., Nucl. Instr. Meth. A 386 (1997) 310 and 348.

[24] H1 Calorimeter Group, B. Andrieu et al., Nucl. Instr. Meth. A 336 (1993) 460.

[25] H1 Calorimeter Group, B. Andrieu et al., Nucl. Instr. Meth. A 350 (1994) 57.

[26] H1 Calorimeter Group, B. Andrieu et al., Nucl. Instr. Meth. A 336 (1993) 499.

[27] H1 Spacal Group, R.D. Appuhn et al., Nucl. Instr. Meth. A 386 (1997) 397.

[28] H1 Collaboration, C. Adloff et al., Eur. Phys. J. C 13 (2000) 609.

[29] U. Bassler and G. Bernardi, Nucl. Instr. Meth. A 426 (1999) 583.

[30] T. Sjöstrand and M. Bengtsson, Comp. Phys. Comm. 43 (1987) 74.

[31] R. Engel, Z. Phys. C 66 (1995) 203;

R. Engel and J. Ranft, Phys. Rev. D 54 (1996) 4244.

[32] CERN, GEANT, detector description and simulation tool, CERN Program Library Long Writeup W 5013 (1994).

[33] H1 Collaboration, C. Adloff et al., Nucl. Phys. B 545 (1999) 3.

[34] H.L. Lai et al., Eur. Phys. J. C 12 (2000) 375.

[35] H1 Collaboration, C. Adloff et al., Nucl. Phys. B 538 (1999) 3.

[36] E.B. Zijlstra and W.L. van Neerven, Nucl. Phys. B 383 (1992) 525.

[37] E. Mirkes and D. Zeppenfeld, Phys. Lett. B 380 (1996) 205.

[38] F. James and M. Roos, Comp. Phys. Comm. 10 (1975) 343.

[39] C. Pascaud and F. Zomer, preprint LAL 95-05 (1995).

[40] M. Botje, Eur. Phys. J. C 14 (2000) 285.

[41] V. Barone, C. Pascaud and F. Zomer, Eur. Phys. J. C 12 (2000) 243. 
[42] H1 Collaboration, C. Adloff et al., Phys. Lett. B 479 (2000) 358.

[43] T. van Ritbergen, J.A.M. Vermaseren and S.A. Larin, Phys. Lett. B 400 (1997) 379.

[44] K.G. Chetyrkin, B. A. Kniehl and M. Steinhauser, Phys. Rev. Lett. 79 (1997) 2184.

[45] S. Bethke, J. Phys. G 26 (2000) R27.

[46] A.D. Martin, R.G. Roberts, W.J. Stirling and R.S. Thorne, Eur. Phys. J. C 14 (2000) 133.

[47] CTEQ Collaboration, H.L. Lai et al., Phys. Rev. D 55 (1997) 1280.

[48] A.D. Martin, R.G. Roberts and W.J. Stirling, Phys. Lett. B 387 (1996) 419.

[49] A.D. Martin, W.J. Stirling and R.G. Roberts, Phys. Lett. B 356 (1995) 89.

[50] J. Huston et al., Phys. Rev. D 58 (1998) 114034.

[51] M. Glück, E. Reya and A. Vogt, Eur. Phys. J. C 5 (1998) 461.

[52] JADE Collaboration and OPAL Collaboration, P. Pfeifenschneider et al., CERN-EP-99175, to appear in Eur. Phys. J. C. 


\section{Tables of Experimental Results}

In the following those jet cross sections are listed which have been used in the QCD analyses to obtain the central results. The numbers of further distributions can be found in [5] or are available on request from the $\mathrm{H} 1$ collaboration.

The Inclusive Jet Cross Section $\mathrm{d}^{2} \sigma_{\text {jet }} /\left(\mathrm{d} E_{T} \mathrm{~d} Q^{2}\right)$

\begin{tabular}{|c||l|}
\hline bin number & corresponding $Q^{2}$ range \\
\hline 1 & $150<Q^{2}<200 \mathrm{GeV}^{2}$ \\
2 & $200<Q^{2}<300 \mathrm{GeV}^{2}$ \\
3 & $300<Q^{2}<600 \mathrm{GeV}^{2}$ \\
4 & $600<Q^{2}<5000 \mathrm{GeV}^{2}$ \\
\hline
\end{tabular}

\begin{tabular}{|c||c|}
\hline bin letter & corresponding $E_{T}$ range \\
\hline a & $7<E_{T}<11 \mathrm{GeV}$ \\
b & $11<E_{T}<18 \mathrm{GeV}$ \\
c & $18<E_{T}<30 \mathrm{GeV}$ \\
d & $30<E_{T}<50 \mathrm{GeV}$ \\
\hline
\end{tabular}

\begin{tabular}{|c|c|c|c|c|c|c|c|c|c|c|c|c|c|c|}
\hline \multicolumn{10}{|c|}{ the inclusive jet cross section $\mathrm{d}^{2} \sigma_{\text {jet }} /\left(\mathrm{d} E_{T} \mathrm{~d} Q^{2}\right)$} & \multicolumn{5}{|c|}{ inclusive $k_{\perp}$ jet algorithm } \\
\hline \multirow[b]{2}{*}{$\begin{array}{l}\text { bin } \\
\text { No. }\end{array}$} & \multirow[b]{2}{*}{$\begin{array}{r}\text { cross } \\
\text { section } \\
\text { (in pb) } \\
\end{array}$} & \multirow[b]{2}{*}{$\begin{array}{r}\text { statistical } \\
\text { uncert. } \\
\text { (in percent) }\end{array}$} & \multirow{2}{*}{\multicolumn{2}{|c|}{$\begin{array}{c}\text { total } \\
\text { uncertainty } \\
\text { (in percent) }\end{array}$}} & \multirow{2}{*}{\multicolumn{2}{|c|}{$\begin{array}{c}\text { uncorrelated } \\
\text { uncertainty } \\
\text { (in percent) }\end{array}$}} & \multirow{2}{*}{\multicolumn{2}{|c|}{$\begin{array}{l}\text { correlated } \\
\text { uncertainty } \\
\text { (in percent) }\end{array}$}} & \multirow{3}{*}{$\begin{array}{r}\text { sing } \\
\begin{array}{r}\text { model dep. } \\
\text { detector corr. } \\
\text { (in percent) }\end{array} \\
+4.7\end{array}$} & \multirow{3}{*}{$\begin{array}{r}\begin{array}{r}\text { contributions to } \\
\text { positron } \\
\text { energy scale } \\
\text { (in percent) }\end{array} \\
0.8\end{array}$} & \multirow{3}{*}{$\begin{array}{r}\text { orrelated unc } \\
\text { positron } \\
\text { polar angle } \\
\text { (in percent) }\end{array}$} & \multirow{2}{*}{\multicolumn{2}{|c|}{$\begin{array}{l}\text { LAr hadr. } \\
\text { energy scale } \\
\text { (in percent) }\end{array}$}} & \multirow{3}{*}{ 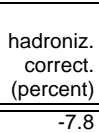 } \\
\hline & & & & & & & & & & & & & & \\
\hline $1 \mathrm{a}$ & $=02.220$ & $7 \pm 2.9$ & 9.6 & -9.5 & 7.6 & $=-7.4$ & 5.9 & -5.9 & & & & $\overline{2.6}$ & -2.6 & \\
\hline $1 \mathrm{~b}$ & 26.084 & \pm 4.4 & 16.3 & -13.9 & 13.6 & -11.3 & 9.0 & -8.0 & \pm 6.3 & 0.8 & 1.6 & 6.0 & -4.4 & -5.0 \\
\hline $1 \mathrm{c}$ & 5.819 & \pm 9.2 & 13.8 & -15.6 & 12.3 & -13.8 & 6.3 & -7.2 & \pm 4.3 & 1.0 & 1.7 & 3.9 & -5.3 & -4.5 \\
\hline $1 \mathrm{~d}$ & 0.719 & $\begin{array}{r} \pm 27.8 \\
\pm\end{array}$ & 34.0 & -35.4 & 32.4 & $\begin{array}{l}-33.4 \\
\end{array}$ & 10.4 & -11.6 & \pm 3.4 & 1.9 & 2.7 & 9.2 & -10.5 & -4.7 \\
\hline $2 a$ & 62.256 & \pm 2.6 & 7.6 & -7.2 & 6.4 & -6.1 & 4.1 & -3.9 & \pm 2.3 & 0.2 & 1.0 & 2.8 & & -8.1 \\
\hline $2 \mathrm{~b}$ & 29.802 & \pm 3.7 & 12.2 & -12.7 & 10.3 & -10.8 & 6.5 & -6.7 & \pm 4.0 & 0.7 & 0.2 & 4.9 & -5.2 & -4.5 \\
\hline $2 \mathrm{c}$ & 6.989 & \pm 7.6 & 16.8 & -14.8 & 14.3 & -12.4 & 8.9 & -8.1 & \pm 6.7 & 0.3 & 1.9 & 5.3 & -3.8 & -4.6 \\
\hline $2 d$ & 0.994 & \pm 20.0 & 28.7 & -33.5 & 26.0 & -29.9 & 12.1 & -15.0 & \pm 8.6 & 2.7 & 1.9 & 7.7 & -11.8 & -4.9 \\
\hline $3 a$ & 61.577 & \pm 2.7 & 5.8 & -6.1 & 4.9 & -5.3 & & & & & 0.8 & 2.0 & -2.2 & -8.1 \\
\hline $3 \mathrm{~b}$ & 35.010 & \pm 2.1 & $\begin{array}{r}5.0 \\
11.9\end{array}$ & $=0.1$ & $\begin{array}{r}4.9 \\
10.1\end{array}$ & -5.3 & $\begin{array}{l}6.0 \\
6.3\end{array}$ & $\begin{array}{l}-5.5 \\
-5.5\end{array}$ & $\begin{array}{l} \pm .2 \\
\pm 3.5\end{array}$ & 0.5 & $\begin{array}{l}1.0 \\
1.4\end{array}$ & 4.8 & $\begin{array}{l}-2.2 \\
-3.6\end{array}$ & -4.0 \\
\hline $3 \mathrm{c}$ & 9.644 & \pm 6.6 & 15.7 & -17.9 & 13.3 & -15.3 & 8.4 & -9.4 & \pm 4.2 & 1.6 & 3.7 & 5.9 & -7.2 & -4.5 \\
\hline $3 d$ & 1.362 & $\begin{array}{l} \pm 20.0 \\
\pm 20\end{array}$ & 36.3 & -29.7 & 32.1 & -26.4 & 17.1 & -13.7 & $\begin{array}{r} \pm 11.4 \\
\end{array}$ & 1.1 & 1.6 & 12.5 & -7.1 & -4.6 \\
\hline $4 \mathrm{a}$ & 46.515 & \pm 3.1 & 7.4 & -6.8 & 6.3 & -5.8 & 3.8 & -3.5 & \pm 0.7 & 0.0 & 1.4 & 3.1 & -2.7 & -9.0 \\
\hline $4 \mathrm{~b}$ & 26.409 & & 8.8 & -8.8 & 7.6 & -7.6 & 4.4 & -4.4 & & 0.6 & 1.5 & 3.5 & & -4.0 \\
\hline $4 \mathrm{c}$ & 11.288 & \pm 6.0 & 11.5 & -11.6 & 10.3 & -10.3 & 5.3 & -5.4 & \pm 2.4 & 0.3 & 0.5 & 4.4 & -4.6 & -3.2 \\
\hline $4 d$ & 1.993 & \pm 15.1 & 27.2 & -23.0 & 24.6 & -21.2 & 11.5 & -8.9 & \pm 1.2 & 0.9 & 1.9 & 11.1 & -8.4 & -3.2 \\
\hline
\end{tabular}

Table 2: Results of the inclusive jet cross section measurement using the inclusive $k_{\perp}$ jet algorithm. The listing includes all experimental uncertainties (as described in section 3.4) which are here separated into the correlated and the uncorrelated part. Since the interpretation of the results (as e.g. in a QCD analysis) does not require the knowledge of the separate contributions to the uncorrelated part of the uncertainties, only the total uncorrelated uncertainty is presented while the single contributions to the correlated uncertainty are listed in extra columns for all sources. The uncertainty from the hadronic energy scale of the Liquid Argon calorimeter is quoted asymmetrically. The left (right) value corresponds to an increase (decrease) of the calibration constants. The uncertainties of the positron energy and the positron polar angle are defined to be symmetric by taking the maximum of the upwards and downwards deviations. The signs are quoted for a positive variation of the corresponding source. Note that only the correlated contribution from these sources is listed. As described in section 3.4 some of these sources contribute also to the uncorrelated uncertainty. The latter contribution is already contained in the (quadratic) sum of all uncorrelated uncertainties. The total correlated uncertainty includes also the contribution of $\pm 1.5 \%$ from the uncertainty in the determination of the luminosity. In the right column we have also included the size of the hadronization corrections as determined by the procedure described in section 5.2 . 
The Dijet Cross Section $d^{2} \sigma_{\mathrm{dijet}} /\left(d \xi d Q^{2}\right)$

\begin{tabular}{|c||c||c|}
\hline bin No. & corresponding $Q^{2}$ range & $\xi$ range \\
\hline $1 \mathrm{a}$ & $5<Q^{2}<10 \mathrm{GeV}^{2}$ & $0.004<\xi<0.01$ \\
$1 \mathrm{~b}$ & & $0.01<\xi<0.025$ \\
$1 \mathrm{c}$ & & $0.025<\xi<0.05$ \\
$1 \mathrm{~d}$ & & $0.05<\xi<0.1$ \\
\hline $2 \mathrm{a}$ & $10<Q^{2}<20 \mathrm{GeV}^{2}$ & $0.004<\xi<0.01$ \\
$2 \mathrm{~b}$ & & $0.01<\xi<0.025$ \\
$2 \mathrm{c}$ & & $0.025<\xi<0.05$ \\
$2 \mathrm{~d}$ & & $0.05<\xi<0.1$ \\
\hline $3 \mathrm{a}$ & $20<Q^{2}<35 \mathrm{GeV}^{2}$ & $0.004<\xi<0.01$ \\
$3 \mathrm{~b}$ & & $0.01<\xi<0.025$ \\
$3 \mathrm{c}$ & & $0.025<\xi<0.05$ \\
$3 \mathrm{~d}$ & & $0.05<\xi<0.1$ \\
\hline $4 \mathrm{a}$ & $35<Q^{2}<70 \mathrm{GeV}^{2}$ & $0.004<\xi<0.01$ \\
$4 \mathrm{~b}$ & & $0.01<\xi<0.025$ \\
$4 \mathrm{c}$ & & $0.025<\xi<0.05$ \\
$4 \mathrm{~d}$ & & $0.05<\xi<0.1$ \\
\hline
\end{tabular}

\begin{tabular}{|c||c||c|}
\hline bin No. & corresponding $Q^{2}$ range & $\xi$ range \\
\hline $5 \mathrm{a}$ & $150<Q^{2}<200 \mathrm{GeV}^{2}$ & $0.009<\xi<0.017$ \\
$5 \mathrm{~b}$ & & $0.017<\xi<0.025$ \\
$5 \mathrm{c}$ & & $0.025<\xi<0.035$ \\
$5 \mathrm{~d}$ & & $0.035<\xi<0.05$ \\
$5 \mathrm{e}$ & & $0.05<\xi<0.12$ \\
\hline $6 \mathrm{a}$ & $200<Q^{2}<300 \mathrm{GeV}^{2}$ & $0.01<\xi<0.02$ \\
$6 \mathrm{~b}$ & & $0.02<\xi<0.03$ \\
$6 \mathrm{c}$ & & $0.03<\xi<0.04$ \\
$6 \mathrm{~d}$ & & $0.04<\xi<0.06$ \\
$6 \mathrm{e}$ & & $0.06<\xi<0.15$ \\
\hline $7 \mathrm{a}$ & $300<Q^{2}<600 \mathrm{GeV}^{2}$ & $0.015<\xi<0.025$ \\
$7 \mathrm{~b}$ & & $0.025<\xi<0.035$ \\
$7 \mathrm{c}$ & & $0.035<\xi<0.045$ \\
$7 \mathrm{~d}$ & & $0.045<\xi<0.065$ \\
$7 \mathrm{e}$ & & $0.065<\xi<0.18$ \\
\hline $8 \mathrm{a}$ & $600<Q^{2}<5000 \mathrm{GeV}^{2}$ & $0.025<\xi<0.045$ \\
$8 \mathrm{~b}$ & & $0.045<\xi<0.065$ \\
$8 \mathrm{c}$ & & $0.065<\xi<0.1$ \\
$8 \mathrm{~d}$ & & $0.1<\xi<0.3$ \\
\hline
\end{tabular}

\begin{tabular}{|c|c|c|c|c|c|c|c|c|c|c|c|c|c|c|}
\hline \multirow{4}{*}{$\begin{array}{l}\text { bin } \\
\text { No. } \\
1 \mathrm{a}\end{array}$} & \multicolumn{5}{|c|}{ the dijet cross section } & \multicolumn{3}{|c|}{$d^{2} \sigma_{\text {dijet }} /\left(d \xi d Q^{2}\right)$} & \multicolumn{6}{|c|}{ inclusive $k_{\perp}$ jet algorithm } \\
\hline & \multirow[b]{2}{*}{$\begin{array}{r}\text { cross } \\
\text { section } \\
\text { (in pb) }\end{array}$} & \multirow[b]{2}{*}{$\begin{array}{r}\text { statistical } \\
\text { uncert. } \\
\text { (in percent) }\end{array}$} & \multirow{2}{*}{\multicolumn{2}{|c|}{$\begin{array}{c}\text { total } \\
\text { uncertainty } \\
\text { (in percent) }\end{array}$}} & \multirow{2}{*}{\multicolumn{2}{|c|}{$\begin{array}{l}\text { uncorrelated } \\
\text { uncertainty } \\
\text { (in percent) }\end{array}$}} & \multirow{2}{*}{\multicolumn{2}{|c|}{$\begin{array}{l}\text { correlated } \\
\text { uncertainty } \\
\text { (in percent) }\end{array}$}} & \multicolumn{5}{|c|}{ single contributions to correlated uncertainty } & \multirow[b]{2}{*}{$\begin{array}{r}\text { hadroniz. } \\
\text { correct. } \\
\text { (percent) }\end{array}$} \\
\hline & & & & & & & & & $\begin{array}{r}\text { model dep. } \\
\text { detector corr. } \\
\text { (in percent) }\end{array}$ & $\begin{array}{r}\text { positron } \\
\text { energy scale } \\
\text { (in percent) }\end{array}$ & $\begin{array}{r}\text { positron } \\
\text { polar angle } \\
\text { (in percent) }\end{array}$ & $\begin{array}{l}\text { Le } \\
\text { ene } \\
\text { (in }\end{array}$ & $\begin{array}{l}\text { adr. } \\
\text { scale } \\
\text { cent) }\end{array}$ & \\
\hline & 19.34 & 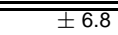 & $\overline{10.6}$ & -10.0 & $\overline{8.5}$ & $\overline{-8.5}$ & 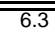 & -5.3 & \pm 2.4 & -0.2 & -3.6 & $\overline{4.9}$ & -3.0 & -6.1 \\
\hline $1 \mathrm{~b}$ & 83.84 & \pm 3.8 & 10.8 & -9.2 & 8.4 & -6.3 & 6.8 & -6.7 & \pm 1.1 & 2.7 & 4.8 & 3.7 & -3.6 & -9.3 \\
\hline $1 \mathrm{c}$ & 47.96 & $\begin{array}{l} \pm \\
\pm\end{array}$ & 6.3 & -10.1 & 4.8 & -8.2 & 4.2 & -5.9 & \pm 3.8 & 0.6 & 2.7 & 2.7 & -3.5 & -5.9 \\
\hline $1 \mathrm{~d}$ & 18.72 & \pm 7.4 & 16.8 & -8.6 & 13.6 & -6.9 & 9.9 & -5.2 & \pm 6.2 & 2.6 & 2.1 & 6.8 & -4.0 & -9.3 \\
\hline $2 a$ & 14.67 & \pm 7.1 & 8.6 & -9.2 & 6.9 & -7.6 & 5.1 & -5.2 & \pm 2.2 & 1.6 & -2.3 & 4.0 & -3.8 & -13.2 \\
\hline $2 b$ & 66.71 & \pm 3.6 & 8.0 & -9.9 & 6.6 & -8.7 & 4.5 & -4.7 & \pm 2.0 & 1.0 & -1.6 & 3.8 & -3.8 & -6.4 \\
\hline $2 c$ & 39.42 & \pm 5.0 & 12.2 & -7.8 & 10.2 & -5.6 & 6.6 & -5.4 & \pm 3.2 & 1.1 & 3.2 & 5.4 & -2.7 & -5.6 \\
\hline $2 d$ & 14.58 & \pm 7.8 & 11.2 & -13.3 & 8.8 & -10.8 & 6.9 & -7.7 & \pm 3.7 & -1.7 & 4.0 & 5.1 & -5.2 & -11.4 \\
\hline $3 a$ & 9.45 & \pm 8.8 & 15.7 & -11.9 & 13.4 & -10.6 & 8.2 & -5.6 & \pm 1.7 & 2.4 & 1.1 & 7.6 & -4.6 & -12.7 \\
\hline $3 b$ & 49.42 & \pm 4.0 & 7.9 & -9.2 & 6.5 & -8.1 & 4.5 & -4.4 & \pm 1.1 & 0.8 & 1.7 & 3.8 & -3.8 & -7.0 \\
\hline $3 c$ & 28.83 & \pm 5.3 & 7.1 & -11.4 & 5.4 & -9.9 & 4.6 & -5.7 & \pm 0.3 & -1.8 & -2.4 & 3.1 & -4.8 & -6.6 \\
\hline $3 d$ & 10.90 & \pm 9.6 & 12.4 & -12.4 & 10.2 & -9.8 & 7.1 & -7.6 & \pm 4.7 & 3.1 & 1.8 & 5.9 & -4.8 & 4.2 \\
\hline $4 a$ & 6.46 & \pm 10.6 & 16.7 & -9.3 & 15.0 & -7.6 & 7.4 & -5.3 & \pm 1.5 & 1.9 & 1.9 & 6.7 & -4.3 & -3.0 \\
\hline $4 b$ & 47.92 & \pm 3.8 & 9.5 & -7.0 & 8.2 & -5.7 & 4.9 & -4.1 & \pm 1.0 & -1.3 & 2.1 & 3.8 & -3.3 & -4.7 \\
\hline $4 \mathrm{c}$ & 31.09 & \pm 4.9 & 6.7 & -9.8 & 5.4 & -8.3 & 3.8 & -5.3 & \pm 2.4 & -1.1 & 1.1 & 3.1 & -4.4 & -5.0 \\
\hline $4 d$ & 12.06 & \pm 8.0 & 13.8 & -9.0 & 11.9 & -7.5 & 7.1 & -5.0 & \pm 0.4 & 0.9 & 2.4 & 6.5 & -4.3 & -11.5 \\
\hline $5 a$ & 4.147 & \pm 11.0 & 24.7 & -27.7 & 19.9 & -22.9 & 14.7 & -15.6 & \pm 13.7 & 1.9 & 1.8 & 4.3 & -6.9 & -6.0 \\
\hline $5 \mathrm{~b}$ & 6.272 & \pm 9.1 & 14.9 & -13.8 & 13.2 & -12.2 & 6.9 & -6.4 & \pm 3.4 & 2.1 & 2.8 & 4.7 & -3.8 & -5.3 \\
\hline $5 c$ & 6.544 & \pm 9.8 & 12.3 & -13.2 & 11.2 & -12.0 & 5.1 & -5.6 & \pm 3.1 & 3.0 & 1.2 & 1.8 & -3.0 & -5.7 \\
\hline $5 d$ & 5.059 & \pm 10.9 & 14.3 & -15.3 & 13.2 & -14.1 & 5.3 & -5.9 & \pm 1.9 & 2.3 & 2.2 & 3.5 & -4.4 & -5.4 \\
\hline $5 \mathrm{e}$ & 4.800 & \pm 11.3 & 18.4 & -17.8 & 15.1 & -14.5 & 10.6 & -10.3 & \pm 7.9 & 3.3 & 5.3 & 2.9 & -1.7 & -6.2 \\
\hline $6 a$ & 6.324 & \pm 8.8 & 13.7 & -18.1 & 12.0 & -16.0 & 6.5 & -8.6 & \pm 3.8 & 3.6 & 1.2 & 3.4 & -6.5 & -6.1 \\
\hline $6 \mathrm{~b}$ & 7.309 & \pm 7.8 & 10.9 & -12.5 & 10.0 & -11.3 & 4.4 & -5.3 & \pm 2.6 & 0.3 & 1.0 & 3.1 & -4.3 & -4.8 \\
\hline $6 \mathrm{c}$ & 6.023 & \pm 8.6 & 11.7 & -11.8 & 10.5 & -10.5 & 5.2 & -5.5 & \pm 1.1 & 1.5 & 3.7 & 2.8 & -3.2 & -5.0 \\
\hline $6 \mathrm{~d}$ & 5.512 & \pm 8.9 & 14.9 & -11.9 & 13.4 & -11.0 & 6.6 & -4.6 & \pm 2.6 & 1.6 & 1.9 & 5.3 & -2.4 & -4.8 \\
\hline $6 \mathrm{e}$ & 4.186 & \pm 10.3 & 17.8 & -19.3 & 14.7 & -16.1 & 10.1 & -10.8 & \pm 8.3 & 3.1 & 3.6 & 3.0 & -4.8 & -7.5 \\
\hline $7 a$ & 5.997 & \pm 9.3 & 12.7 & -15.4 & 11.9 & -14.4 & 4.5 & -5.6 & \pm 1.5 & 0.9 & 1.0 & 3.8 & -5.1 & -5.4 \\
\hline $7 b$ & 7.006 & \pm 8.3 & 11.3 & -12.4 & 10.1 & -11.1 & 5.0 & -5.6 & \pm 3.5 & 0.4 & 2.1 & 2.4 & -3.4 & -4.8 \\
\hline $7 \mathrm{c}$ & 6.104 & \pm 9.0 & 16.0 & -11.9 & 14.5 & -11.1 & 6.9 & -4.5 & \pm 1.2 & 2.2 & 1.0 & 6.1 & -3.3 & -4.5 \\
\hline $7 d$ & 7.249 & \pm 8.1 & 10.1 & -10.4 & 9.0 & -9.2 & 4.7 & -4.9 & \pm 1.9 & 0.6 & 3.8 & 1.2 & -1.9 & -6.2 \\
\hline $7 e$ & 6.082 & \pm 8.8 & 14.7 & -14.3 & 13.1 & -12.7 & 6.7 & -6.5 & \pm 3.0 & 1.2 & 2.4 & 5.1 & -4.9 & -5.8 \\
\hline $8 a$ & 6.077 & \pm 9.4 & 15.0 & -12.6 & 13.6 & -11.6 & 6.3 & -5.0 & \pm 2.3 & 0.6 & 2.0 & 5.2 & -3.5 & -6.2 \\
\hline $8 b$ & 6.759 & \pm 8.5 & 13.3 & -11.9 & 11.7 & -10.5 & 6.3 & -5.6 & \pm 3.8 & 2.3 & 1.9 & 3.8 & -2.4 & -5.6 \\
\hline $8 \mathrm{c}$ & 8.305 & \pm 8.0 & 11.0 & -10.2 & 10.2 & -9.5 & 4.1 & -3.5 & \pm 0.6 & 0.9 & 1.0 & 3.6 & -2.8 & -4.8 \\
\hline $8 d$ & 7.520 & \pm 8.2 & 10.2 & -11.2 & 9.6 & -10.4 & 3.3 & -4.1 & \pm 0.4 & 1.3 & 0.3 & 2.7 & -3.5 & -5.7 \\
\hline
\end{tabular}

Table 3: Results of the dijet cross section measurement at high $Q^{2}$ using the inclusive $k_{\perp}$ jet algorithm. The presentation is as in table 2 . 


\section{Results of the QCD Analysis}

\section{$E_{T}$ Dependence of $\alpha_{s}$}

\begin{tabular}{|c|c|c|c|c|}
\hline \multicolumn{5}{|c|}{$\begin{array}{cc}E_{T} \text { dependence of } \alpha_{s}\left(E_{T}\right) & \left(\mu_{r}=E_{T}\right) \\
\text { inclusive jet cross section }- \text { inclusive } k_{\perp} \text { algorithm }\end{array}$} \\
\hline average $E_{T}$ of data point: & $\sqrt{70} \mathrm{GeV}$ & $\sqrt{200} \mathrm{GeV}$ & $\bar{~} \sqrt{500 \mathrm{GeV}}$ & $\bar{~} \sqrt{1500} \mathrm{GeV}$ \\
\hline$\overline{\alpha_{s}\left(E_{T}\right)=}$ & 0.1940 & $\overline{0.1636}$ & 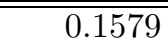 & 0.1507 \\
\hline total uncertainty & $\begin{array}{l}+0.0157 \\
-0.0145\end{array}$ & $\begin{array}{l}+0.0145 \\
{ }_{-0.0136}\end{array}$ & $\begin{array}{l}+0.0162 \\
{ }_{-0.0167}\end{array}$ & $\begin{array}{l}+0.0308 \\
{ }_{-0.0315}\end{array}$ \\
\hline $\begin{array}{l}\text { exp. } \\
\text { theor. } \\
\text { pdf }\end{array}$ & $\begin{array}{l}+0.00821 \\
-0.0081 \\
+-0.00103 \\
+0.0103 \\
+0.0092 \\
-0.0061 \\
+0.007\end{array}$ & $\begin{array}{l}+0.01064 \\
-0.0104 \\
+0.0077 \\
-0.0081 \\
+0.0040 \\
-0.0033\end{array}$ & $\begin{array}{l}+0.0124 \\
-0.0123 \\
+0.0091 \\
-0.0086 \\
+0.0051 \\
-0.0072 \\
\end{array}$ & $\begin{array}{l}+0.0264 \\
-0.0244 \\
-0.0113 \\
-0.0088 \\
+0.0110 \\
-0.0179\end{array}$ \\
\hline$\alpha_{s}\left(M_{Z}\right)=$ & 0.1211 & 0.1174 & 0.1227 & 0.1292 \\
\hline total uncertainty & $\begin{array}{l}+0.0058 \\
-0.0056\end{array}$ & $\begin{array}{l}+0.0068 \\
-0.0070\end{array}$ & $\begin{array}{l}+0.0094 \\
-0.0101\end{array}$ & $\begin{array}{l}+0.0218 \\
-0.0233\end{array}$ \\
\hline $\begin{array}{l}\text { exp. } \\
\text { theor. } \\
\text { pdf }\end{array}$ & $\begin{array}{l}+0.0031 \\
-0.0031 \\
+0.0035 \\
-0.0040 \\
+0.0035 \\
-0.0023 \\
-0.0023\end{array}$ & $\begin{array}{l}+0.0052 \\
-0.0053 \\
+0.0038 \\
-0.0043 \\
+0.0020 \\
-0.0017\end{array}$ & $\begin{array}{l}+0.0072 \\
-0.0074 \\
+0.0053 \\
-0.0054 \\
+0.0030 \\
-0.0042\end{array}$ & $\begin{array}{l}+0.0182 \\
+0.0182 \\
+0.0067 \\
-0.0067 \\
-0.0129 \\
-0.0129\end{array}$ \\
\hline
\end{tabular}

Table 4: The $\alpha_{s}$ results from the fits presented in section 5.3. Displayed are the fit results of $\alpha_{s}\left(E_{T}\right)$ at different $E_{T}$ (top) and the corresponding values extrapolated to $\mu_{r}=M_{Z}$ (bottom) together with the different contributions to the uncertainty.

\section{The Gluon Density in the Proton}

\begin{tabular}{|c|c|c|c|c|}
\hline \multicolumn{4}{|c|}{$\begin{array}{rc}\text { parameterized by } & x G(x)=A x^{b}(1-x)^{c}(1+d x) \text { in } 0.01<x<0.1 \\
\text { central result: } & \mathrm{A}=0.503 ; \mathrm{b}=-0.5935 ; \mathrm{c}=4.70 ; \mathrm{d}=-0.55\end{array}$} & \\
\hline $\log _{10}(x)$ & $x=$ & exp. & theor. & from $\Delta \alpha_{s}\left(M_{Z}\right)$ \\
\hline-2.0 & $7.35_{-1.25}^{+1.34}$ & $\begin{array}{l}+0.93 \\
-0.94\end{array}$ & $\begin{array}{l}+0.77 \\
{ }_{-0.71}\end{array}$ & \\
\hline-1.9 & $6.32_{-0.98}^{+1.04}$ & $\begin{array}{l}-0.94 \\
+0.64 \\
-0.65\end{array}$ & $\begin{array}{l}-0.11 \\
-0.65 \\
-0.63\end{array}$ & $\begin{array}{l}-0.45 \\
+0.49 \\
-0.37\end{array}$ \\
\hline-1.8 & $5.42_{-0.77}^{+0.82}$ & $\begin{array}{l}+0.45 \\
{ }_{-0.45}\end{array}$ & $\begin{array}{l}-0.63 \\
-0.54 \\
-0.54\end{array}$ & $\begin{array}{l}-0.35 \\
+0.42 \\
-0.32\end{array}$ \\
\hline-1.7 & $4.63_{-0.63}^{+0.65}$ & $\begin{array}{l}{ }_{-0.32}^{+0.32} \\
-0.32\end{array}$ & $\begin{array}{l}-0.04 \\
{ }_{-0.46}^{0.44}\end{array}$ & $\begin{array}{l}-0.36 \\
-0.28\end{array}$ \\
\hline-1.6 & $3.93_{-0.52}^{+0.54}$ & ${ }_{-0.25}^{+0.26}$ & $\begin{array}{l}+0.36 \\
-0.38\end{array}$ & $\begin{array}{l}-0.20 \\
+0.24 \\
-0.24\end{array}$ \\
\hline-1.5 & $3.31_{-0.43}^{+0.45}$ & $\begin{array}{l}+0.23 \\
{ }_{-0.21}\end{array}$ & $\begin{array}{l}+0.28 \\
-0.31\end{array}$ & $\begin{array}{l}+0.25 \\
-0.20\end{array}$ \\
\hline-1.4 & $2.76_{-0.37}^{+0.37}$ & +0 & +0.22 & +0.21 \\
\hline-1.3 & $2.27_{-0.30}^{+0.31}$ & $\begin{array}{l}+0.19 \\
+0.18\end{array}$ & $\begin{array}{l}+0.18 \\
-0.19\end{array}$ & $\begin{array}{l}+0.17 \\
+0.14\end{array}$ \\
\hline-1.2 & $1.84_{-0.25}^{+0.26}$ & ${ }_{-0}^{+0}$ & $\begin{array}{l}+0.13 \\
+0.14\end{array}$ & $\begin{array}{l}-0.14 \\
+0.14 \\
-0.12\end{array}$ \\
\hline-1.1 & $1.47_{-0.20}^{+0.22}$ & ${ }_{-0}^{+0}$ & $\begin{array}{l}-0 . \\
+0 .\end{array}$ & $\begin{array}{l}-0.11 \\
+0.09\end{array}$ \\
\hline-1.0 & $1.14_{-0.17}^{+0.19}$ & $\begin{array}{l}-0.1 \\
+0.1\end{array}$ & $\begin{array}{l}-0.11 \\
{ }_{-0.10}^{+0.08}\end{array}$ & $\begin{array}{l}-0.09 \\
{ }_{-0.07}^{-0.08}\end{array}$ \\
\hline
\end{tabular}

Table 5: The gluon density in the proton from the fit in section 5.4. Displayed are the central results and the total uncertainties of the gluon density at eleven values of $x$ in the interval $0.01 \leq x \leq 0.1$ together with the different contributions to the uncertainty. Also shown are the parameters $A, b, c, d$ of the central result. 